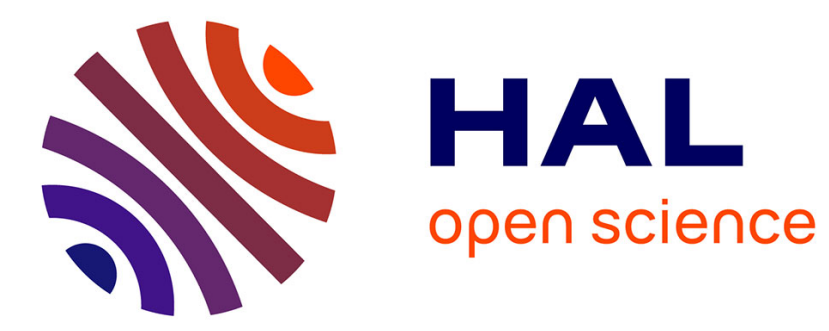

\title{
Effects of uranium on the metabolism of zebrafish, Danio rerio
}

Starrlight Augustine, Béatrice Gagnaire, Christelle Adam-Guillermin, Sebastiaan A.L.M. Kooijman

\section{- To cite this version:}

Starrlight Augustine, Béatrice Gagnaire, Christelle Adam-Guillermin, Sebastiaan A.L.M. Kooijman. Effects of uranium on the metabolism of zebrafish, Danio rerio. Aquatic Toxicology, 2012, 118- 119, pp.9- 26. 10.1016/j.aquatox.2012.02.029 . hal-00760075

\section{HAL Id: hal-00760075 https://hal.science/hal-00760075}

Submitted on 3 Dec 2012

HAL is a multi-disciplinary open access archive for the deposit and dissemination of scientific research documents, whether they are published or not. The documents may come from teaching and research institutions in France or abroad, or from public or private research centers.
L'archive ouverte pluridisciplinaire HAL, est destinée au dépôt et à la diffusion de documents scientifiques de niveau recherche, publiés ou non, émanant des établissements d'enseignement et de recherche français ou étrangers, des laboratoires publics ou privés. 


\title{
Effects of uranium on the metabolism of zebrafish, Danio rerio
}

\author{
Starrlight Augustine ${ }^{\dagger 1}$, Béatrice Gagnaire ${ }^{\dagger}$, Christelle Adam-Guillermin ${ }^{\dagger}$ and Sebastiaan \\ A.L.M. Kooijman ${ }^{\ddagger}$ \\ † Laboratory of Radionuclide Ecotoxicology, PRP-ENV/SERIS/LECO, Institute of Radioprotection and \\ Nuclear Safety (IRSN), Caradache, Building 186, BP3, 13115 St-Paul-lez-Durance Cedex, France \\ $\ddagger$ Department of Theoretical Biology, Vrije Universiteit, de Boelelaan 1087, 1081 HV Amsterdam, the \\ Netherlands
}

Author's version before typesetting by Elsevier

Figures are designed to be reproduced in colour on the web and in black-and-white in print.

Starrlight Augustine: e-mail: starr-light.augustine@irsn.fr, tel: 0033(0)442199413

Béatrice Gagnaire: beatrice.gagnaire@irsn.fr, tel: 0033(0)442199401

Christelle Adam-Guillermin: christelle.adam-guillermin@irsn.fr, tel: 0033(0)442199493

Sebastiaan A. L. M. Kooijman: bas.kooijman@vu.nl, tel: 0031(0)205987130

\footnotetext{
${ }^{1}$ Corresponding author
} 


\begin{abstract}
The increasing demand for nuclear energy results in heightened levels of uranium (U) in aquatic systems which present a potential health hazard to resident organisms. The aim of this study was to mechanistically assess how chronic exposure to environmentally relevant concentrations of U perturbs the complex interplay between feeding, growth, maintenance, maturation and reproduction throughout the life-cycle of an individual. To this end we analysed literature-based and original zebrafish toxicity data within a same mass and energy balancing conceptual framework. U was found to increase somatic maintenance leading to inhibition of spawning as well as increase hazard rate and costs for growth during the early life stages. The fish's initial conditions and elimination through reproduction greatly affected toxico-kinetics and effects. We demonstrate that growth and reproduction should be measured on specific individuals since mean values were hardly interpretable. The mean food level differed between experiments, conditions and individuals. This last 'detail' contributed substantially to the observed variability by its combined effect on metabolism, toxic effects and toxico-kinetics. The significance of this work is that we address exactly how these issues are related and derive conclusions which are independent of experimental protocol and coherent with a very large body of literature on zebrafish eco-physiology.
\end{abstract}

Key words: Dynamic Energy Budget theory, growth, metabolism, reproduction, uranium, zebrafish 


\section{Introduction}

Uranium (U) is an ubiquitous element in the environment but natural concentrations in aquatic systems are increased in response to human activities linked to the nuclear fuel cycle (e.g. Fernandes et al., 1995; Jurgens et al., 2010; Uralbekov et al., 2011; Villa et al., 2011). A renewed international use of nuclear power as energy source (Nuclear Energy Agency, 2008) and increased public awareness of long term impacts of environmental pollution motivate the study of uranium's toxicity to living organisms. The objective of this study is to mechanistically assess how chronic exposure to environmentally relevant concentrations of U perturbs the complex interplay between feeding, growth, maintenance, maturation and reproduction throughout the life-cycle of an individual. Unfortunately, many toxicity tests concern organisms at particular life stages and experiment duration may be short relative to total lifespan meaning that our method must be able to extrapolate effects across life stages. An additional complex issue for extrapolation purposes is accounting for the relationship between $U$ speciation and water hardness and $\mathrm{pH}$ in combination with toxic effects (Fortin et al., 2004, 2007).

We first compiled four literature-based studies where zebrafish, Danio rerio were chronically exposed to water-borne depleted U. Second, we designed an experiment to mechanistically assess toxicological effects of $U$ on reproductive physiology of zebrafish. Behavioural effects might result from an impairment of sensory organs and inhibit spawning or even oocyte maturation but not the actual mass investment in reproduction. On the other hand allocation to reproduction might be modified in response to perturbations of energy allocation to physiological processes such as growth. The advantage of working with these particular studies is twofold. First, zebrafish are extremely well studied model organisms (Laale, 1977; Hill et al., 2005). Second, the chemical composition of $U$ used for the exposure in combination with the $\mathrm{pH}$, chemical composition and temperature of the medium were similar across all five experiments (table A.1, Online Appendix A).

Differences in effects and accumulation observed across all of the experiments may be (partially) explained by differences in size and growth rate. Therefore there is a need to specify energetics (growth and reproduction) in combination with toxico-kinetics (dynamics of internal concentration) in a unique theoretical framework (Jager et al., 2006; Jager and Klok, 2010; Jager and Zimmer, 2012). A well worked out quantitative theory on metabolic organisation, Dynamic Energy Budget (DEB) theory, provides the toolkit necessary to address this problem (Kooijman, 2010). The theory provides a standard DEB model applicable to all animals (Kooijman, 2010, Chap.2); the model is mechanistic so each parameter quantifies a single metabolic process. The DEB model fully specifies all mass and energy fluxes (Sousa et al., 2010) over the entire life-cycle. 7 metabolic processes are defined: assimilation, mobilization, allocation, synthesis of structure, somatic maintenance, maturation, maturity maintenance (fig. 1A). For our specific purpose we needed to extend the standard model with a module for the preparation of batches of eggs from material in the reproduction buffer (fig. 1B).

A DEB model was previously fully parametrized to zebrafish; predicted development, growth and reproduction closely matched observations published in numerous studies ranging from the 1960's to the present day (Augustine et al., 2011a) and covering the full life-cycle. The zebrafish DEB model presents coherence between observations made with different individuals in combination with different temperature and feeding protocols and further provides estimates for 
all of the standard DEB model parameter values (table 1). We consider that this model provides a correct quantitative characterization of the baseline metabolism of (control) zebrafish. The model can be extended with a one compartment toxico-kinetics module (Kooijman and van Haren, 1990) which specifies the dynamics of the internal concentration, expressed in $\left[M_{Q}\right]$ (nmol $\mathrm{U} \mathrm{cm}^{-3}$ ), which then gets treated as a state variable. In line with DEB theory we assume three ranges of $\left[M_{Q}\right]$ for all compounds: too little, enough and too much. Uranium is a nonessential element, and accordingly, our focus is on what happens in the neighbourhood of the too much range which occurs just above the no effect internal concentration $\left[M_{Q 0}^{*}\right]\left(\mathrm{nmol} \mathrm{U} \mathrm{cm}{ }^{-3}\right)$ for process $*$. Complex patterns of organism level effects which occur just above $\left[M_{Q 0}^{*}\right]$ can be captured by the modification of a single parameter value; this modified parameter quantifies process $*$. If the (internal) concentration further increases, more and more parameters can become affected. From an environmental risk assessment perspective, the interest is on how organism level effects observed in the neighbourhood of $\left[M_{Q 0}^{*}\right]$ translates into long term effects on populations and ecosystems. Thus the identification of the first process which is affected in the presence of uranium is the primary concern of this analysis (Kooijman and Bedaux, 1996; Kooijman, 2010; Jager et al., 2010).

We test the hypothesis that the results across studies are coherent when accounting for effects of energetics (differences in feeding, size, initial amount of reproductive material, elimination through reproduction) on toxico-kinetics. We first describe the zebrafish DEB model and specify the five modules needed to conduct this study. Second we compare model predictions with data. We finish with a discussion on insight gained through the comparative analysis of all data sets using the same coherent framework.

\section{DEB model}

We refer to Augustine et al. (2011a) for the full description of the zebrafish DEB model. Briefly, the assimilation energy flux $\dot{p}_{A}=f\left\{\dot{p}_{A m}\right\} L^{2}\left(\mathrm{~J} \mathrm{~d}^{-1}\right)$ quantifies energy from food assimilated into reserve $E$, with $f$ the scaled functional response and $L$ the structural length and $\left\{\dot{p}_{A m}\right\}$ the maximum surface-area linked assimilation $\left(\mathrm{J} \mathrm{cm}^{-2} \mathrm{~d}^{-1}\right) . L$ is the cubic root of structural volume $V$ (table 2). $f$ is the ratio of actual ingestion over the maximum ingestion possible for an individual of size $L$, also called ingestion level. Differences in feeding across experiments and/ or individuals are quantified by differences in $f$. Dynamics of the four state variables $(V$, $E$, maturity $E_{H}$ and cumulated energy invested in reproduction $E_{R}$ ) are specified in table B.2, Online Appendix B. A fraction $\kappa$ of mobilised reserve is allocated to somatic maintenance and growth, the remaining fraction to maturity maintenance and maturation. The energy conductance, $\dot{v}\left(\mathrm{~cm} \mathrm{~d}^{-1}\right)$, controls reserve mobilization. Somatic maintenance, $\dot{p}_{M}=\left[\dot{p}_{M}\right] V(\mathrm{~J}$ $\left.\mathrm{d}^{-1}\right)$, always takes priority over growth $(d V / d t)$; as does maturity maintenance, $\dot{p}_{J}=\dot{k}_{J} E_{H}(\mathrm{~J}$ $\left.\mathrm{d}^{-1}\right)$ over maturation $d E_{H} / d t\left(\mathrm{~J} \mathrm{~d}^{-1}\right)$. The maturity maintenance coefficient $\dot{k}_{J}$ and volumelinked somatic maintenance $\left[\dot{p}_{M}\right]$ are model parameters (table 1$)$.

Transitions from one life-stage to another occur at fixed maturity levels. Four life stages are defined: embryo, juvenile (juv) I, juv II and adult. Embryo, juv II and adult all grow isomorphically (no change in shape, so surface area is proportional to volume ${ }^{2 / 3}$ ), but juv I grows V1-morphically (surface area is proportional to volume). The latter gives metabolic 


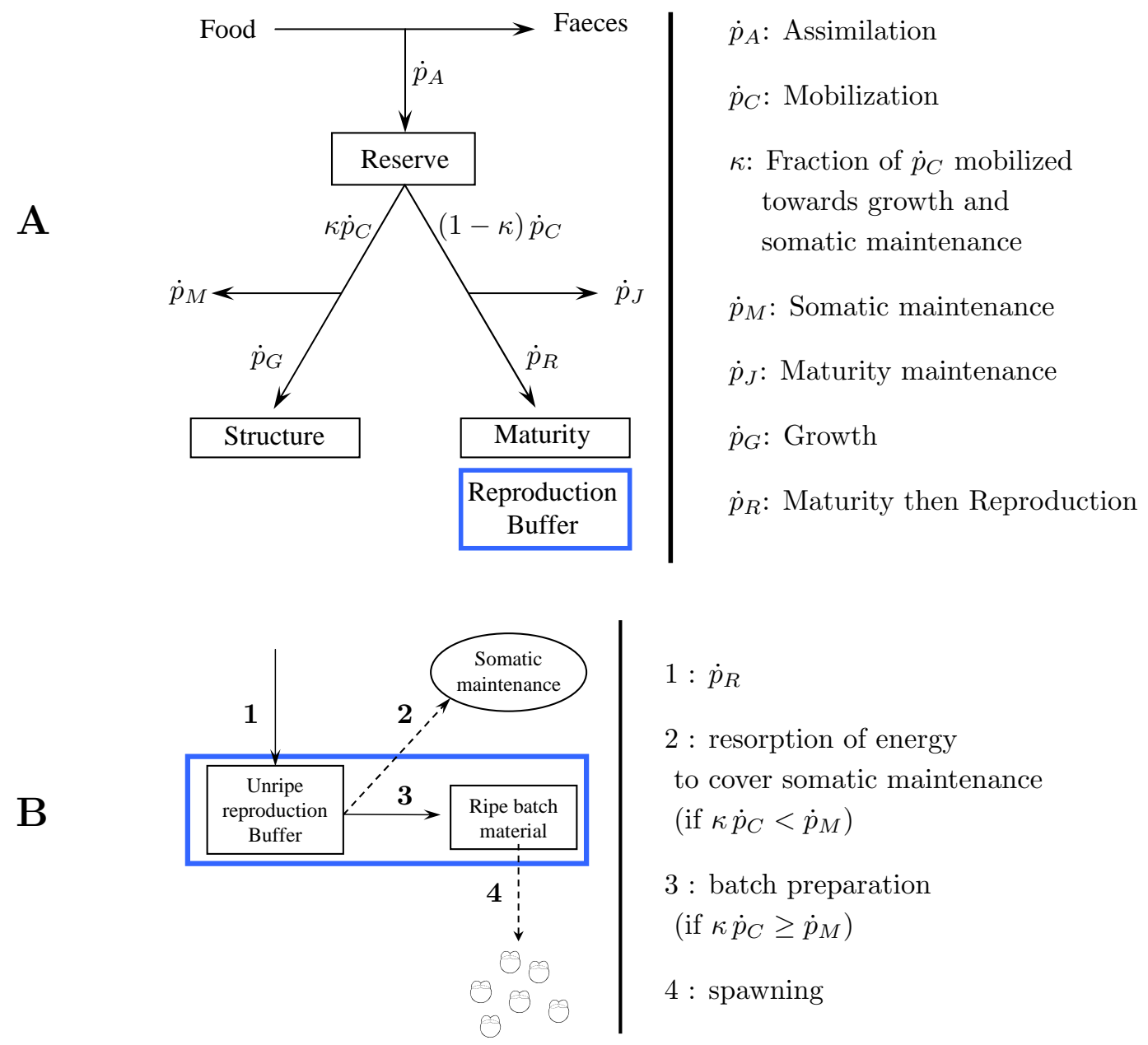

Figure 1: Conceptual organisation of animal metabolism as defined by DEB theory (Kooijman, 2010) and modified from Pecquerie et al. (2009). Full arrows: energy fluxes $\left(\mathrm{J} \mathrm{d}^{-1}\right)$; dotted arrows: energy $(\mathrm{J})$; boxes: state variables of the system; circle: energy sink. Embryo: $\dot{p}_{A}=0$; birth: assimilation is switched on; puberty: allocation to maturity $E_{H}$ stops and allocation to reproduction $E_{R}$ starts. Energy allocated to reproduction accumulates in the reproduction buffer and is emptied at spawning. A: Overview of metabolic organisation. B: reproduction buffer handling rules. 
Table 1: Parameters affecting state variables at reference temperature $\left(20^{\circ} \mathrm{C}\right)$. When adult values differ from that of embryos and early juveniles they are specified in parentheses. g: gram dry mass unless specified otherwise. See tables C.4 and C.3, Online Appendix C for values of toxicity and buffer handling rule parameters used in this study.

\begin{tabular}{|c|c|c|c|}
\hline symbol & definition & unit & value \\
\hline \multicolumn{4}{|c|}{ Toxicity parameters (this study): } \\
\hline$k_{e}$ & elimination rate & $\mathrm{d}^{-1}$ & \\
\hline$P_{V d}$ & biomass / environmental U partition coefficient & $1 \mathrm{~cm}^{-3}$ & \\
\hline$P_{E V}$ & reserve/ structure partition coefficient & $\mathrm{mol} \mathrm{mol}{ }^{-1}$ & \\
\hline$\left[M_{Q 0}^{*}\right]$ & $\begin{array}{l}\text { no effect internal concentration for processes } * \text {, } \\
\text { with } * \in[G, M, A] \text { (see text) }\end{array}$ & $\mathrm{nmol} \mathrm{cm} \mathrm{cm}^{3}$ & \\
\hline$\left[M_{Q T}^{*}\right]$ & $\begin{array}{l}\text { tolerance concentration for processes }{ }^{*}, \text { with } \\
* \in[G, M, A] \text { (see text) }\end{array}$ & $\mathrm{nmol} \mathrm{cm}{ }^{3}$ & \\
\hline$\left[M_{Q 0}^{\mathrm{S}}\right]$ & no effect internal concentration for survival & $\mathrm{nmol} \mathrm{cm} \mathrm{cm}^{3}$ & \\
\hline$\dot{b}_{\dagger}$ & killing rate & $\begin{array}{l}\mathrm{cm}^{3} \mathrm{nmol}^{-1} \\
\mathrm{~d}^{-1}\end{array}$ & \\
\hline$\dot{h}_{\text {acc }}$ & hazard due to accidents & $\mathrm{d}^{-1}$ & \\
\hline \multicolumn{4}{|c|}{ Reproduction buffer handling rules (this study): } \\
\hline$\left[E_{B}\right]$ & energetic density of batch & $\mathrm{J} \mathrm{cm}^{-3}$ & \\
\hline$t_{b}$ & time of initiation of batch preparation & $\mathrm{d}$ & \\
\hline \multicolumn{4}{|c|}{ Starvation: } \\
\hline$\kappa_{G}$ & growth conversion efficiency & - & $\frac{\bar{\mu}_{V} d_{V}}{\left[E_{G}\right] w_{V}}$ \\
\hline$\dot{k}_{J}^{\prime}$ & specific maturity decay & $\mathrm{d}^{-1}$ & 0 \\
\hline \multicolumn{4}{|c|}{ DEB model parameters estimated in Augustine et al. (2011a): } \\
\hline$T_{A}$ & Arrhenius temperature & $\mathrm{K}$ & 3000 \\
\hline$\left\{\dot{p}_{A m}\right\}$ & maximum surface area-specific assimilation rate & $\mathrm{J} \mathrm{d}^{-1} \mathrm{~cm}^{-2}$ & $246.3(775.8)^{\mathrm{a}}$ \\
\hline$\dot{v}$ & energy conductance & $\mathrm{cm} \mathrm{d}^{-1}$ & $0.03(0.09)^{\mathrm{a}}$ \\
\hline$\kappa$ & allocation fraction to soma & - & 0.437 \\
\hline$\kappa_{R}$ & reproduction efficiency & - & 0.95 \\
\hline$\left[\dot{p}_{M}\right]$ & volume-specific somatic maintenance costs & $\mathrm{J} \mathrm{d}^{-1} \mathrm{~cm}^{-3}$ & 500.9 \\
\hline$\dot{k}_{J}$ & maturity maintenance rate & $\mathrm{d}^{-1}$ & 0.0166 \\
\hline$\left[E_{G}\right]$ & cost of synthesis of a unit of structure & $\mathrm{J} \mathrm{cm}^{-3}$ & 4652 \\
\hline$E_{H}^{b}$ & cum. energy invested in $E_{H}$ at birth & $\mathrm{J}$ & 0.54 \\
\hline$E_{H}^{j}$ & cum. energy invested in $E_{H}$ at metamorphosis & $\mathrm{J}$ & 19.66 \\
\hline$E_{H}^{p}$ & cum. energy invested in $E_{H}$ at puberty & $\mathrm{J}$ & 2062 \\
\hline$d_{V}$ & density of structure & $\mathrm{g} \mathrm{cm}^{-3}$ & 0.2 \\
\hline $\bar{\mu}_{E}$ & chemical potential of $E$ & $\mathrm{~J} \mathrm{~mol}^{-1}$ & $5.0010^{5}$ \\
\hline $\bar{\mu}_{V}$ & chemical potential of $V$ & $\mathrm{~J} \mathrm{~mol}^{-1}$ & $5.0010^{5}$ \\
\hline$w_{E}$ & molar weight of reserve & $\mathrm{g} \mathrm{mol}-1$ & 23.9 \\
\hline$w_{V}$ & molar weight of structure & $\mathrm{g} \mathrm{mol} \mathrm{m}^{-1}$ & 23.9 \\
\hline$\delta_{M}$ & shape coefficient & - & $0.1128(0.1325)$ \\
\hline
\end{tabular}

${ }^{\text {a }}$ assuming $f=1$. 
Table 2: DEB model variables and observable quantities. Parameters used for linking observable quantities to state variables can be found in table 1. Dynamics of state variables are summed up in table B.2, Online Appendix B.

\begin{tabular}{|c|c|c|c|}
\hline symbol & meaning & unit & equations \\
\hline \multicolumn{4}{|c|}{ state variables: } \\
\hline$c_{d}$ & $\begin{array}{l}\text { environmental } \\
\text { concentration of } U\end{array}$ & $\mathrm{nM}$ & \\
\hline$V$ & structure & $\mathrm{cm}^{3}$ & \\
\hline$L$ & structural length & $\mathrm{cm}$ & $V^{1 / 3}$ \\
\hline$E$ & reserve & $\mathrm{J}$ & \\
\hline$[E]$ & reserve density & $\mathrm{J} \mathrm{cm}^{-1}$ & {$[E]=E / V$} \\
\hline$E_{R}^{0}$ & $\begin{array}{l}\text { unripe reproduction } \\
\text { buffer }\end{array}$ & $\mathrm{J}$ & \\
\hline$E_{R}^{1}$ & ripe batch material & $\mathrm{J}$ & \\
\hline$E_{R}$ & reproduction buffer & $\mathrm{J}$ & $E_{R}=E_{R}^{0}+E_{R}^{1}$ \\
\hline$E_{H}$ & maturity & $\mathrm{J}$ & \\
\hline$\left[M_{Q}\right]$ & $\begin{array}{l}\text { internal concentration of } \\
\mathrm{U}\end{array}$ & $\mathrm{nmol} \mathrm{cm} \mathrm{cm}^{-3}$ & \\
\hline \multicolumn{4}{|c|}{ how observable quantities relate to state variables: } \\
\hline$L_{f}$ & $\begin{array}{l}\text { total length (snout to tip } \\
\text { of caudal fin) }\end{array}$ & $\mathrm{cm}$ & $L_{f}=L / \delta_{M}$ \\
\hline$W_{d}$ & dry mass & g & $W_{d}=d_{V} L^{3}+w_{E}\left(E+E_{R}\right) / \bar{\mu}_{E}$ \\
\hline$W_{d}^{0}$ & egg dry mass & $\mathrm{g}$ & $W_{d}^{0}=w_{E} E_{0} / \bar{\mu}_{E}$ \\
\hline$W_{w}$ & wet mass & g & $W_{w}=6 W_{d}$ \\
\hline$\left\langle M_{Q}\right\rangle_{d}$ & $\begin{array}{l}\text { concentration per unit } \\
\text { dry mass }\end{array}$ & $\mathrm{nmol} \mathrm{U/g}$ & {$\left[M_{Q}\right] V / W_{d}$} \\
\hline$\left\langle M_{Q}\right\rangle_{w}$ & $\begin{array}{l}\text { concentration per unit } \\
\text { wet mass }\end{array}$ & $\mathrm{nmol} \mathrm{U/g}$ & {$\left[M_{Q}\right] V / W_{w}$} \\
\hline$N$ & number of eggs & $\#$ & $N=\left[E_{B}\right] V / E_{0}$ \\
\hline
\end{tabular}


acceleration. Birth $\left(E_{H}=E_{H}^{b}\right)$ marks the end of the embryo phase and beginning of juv I; external assimilation is switched on and growth is V1-morphic. $f=0$ during the embryonic stage. Implication of V1-morphy are that $\left\{\dot{p}_{A m}\right\}$ and $\dot{v}$ increase proportional to $L$ (Kooijman et al., 2011). In practice both $\left\{\dot{p}_{A m}\right\}$ and $\dot{v}$ are multiplied by a shape correction function (Eq. (B.5), Online Appendix B). Metamorphosis $\left(E_{H}=E_{H}^{j}\right)$ marks the end of juv I and beginning of juv II; adult values of $\left\{\dot{p}_{A m}\right\}$ and $\dot{v}$ are reached and isomorphic growth ensues. Puberty $\left(E_{H}=E_{H}^{p}\right)$ marks the onset of adulthood. $E_{H}^{b}, E_{H}^{j}$ and $E_{H}^{p}$ are three life stage parameters (table 1 ). Initial $L, E, E_{H}$ and $E_{R}$ need to be specified for each adult.

Several processes interact simultaneously in observable quantities such as growth, measured as incremental differences in physical length $\left(L_{f}\right)$ and/or mass, or reproduction measured as number of eggs produced. Auxiliary theory (Kooijman et al., 2008; Lika et al., 2011a) relates observable quantities, $L_{f}, W_{d}$ (dry mass), $W_{d}^{0}$ (egg dry mass), $W_{w}$ (wet mass) and $N$ (\# eggs), to DEB model state variables (table 2). $L$ is taken proportional to some well chosen physical length $L_{f}$ such that: $L=\delta_{M} L_{f}$, with $\delta_{M}$ the shape coefficient.

We incorporate five modules into the zebrafish DEB model which further specify toxicokinetics (Kooijman and van Haren, 1990) and how effects on metabolism relate to internal concentration (toxico-dynamics) (Kooijman and Bedaux, 1996), temperature effects on metabolic rates (Kooijman, 2010, Chap.1), the frequency of spawning events in combination with \# of eggs per spawn as defined by Pecquerie et al. (2009) (reproduction buffer handling rules in fig. 1B) and starvation rules (Augustine et al., 2011b). The five modules are introduced in the following paragraphs. The modules do not modify the dynamics of state variables $E, V, E_{H}$ or $E_{R}$ (table B.2, Online Appendix B).

\subsection{Temperature affects metabolic rates}

Parameter values given in table 1 affect state variables at $20^{\circ} \mathrm{C}$. We assume that the effect of temperature on all biological rates is well captured by the Arrhenius relationship (Kooijman, 2010, Eqn. 1.2 pp.17), quantified by the Arrhenius temperature $T_{A}$ (table 1 ).

\subsection{Buffer handling rules}

Allocation to reproduction, $\dot{p}_{R}$ is a continuous process whereby energy/mass cumulates in the reproduction buffer $E_{R}$. Spawning on the other hand, represents a discontinuous process whereby energy/mass (and U) is lost during each event. Reproduction buffer handling rules specify the dynamics of spawning and play an important role in the dynamics of total dry mass $W_{d}$. Buffer handling rules were previously specified for a multiple batch spawner of indeterminate fecundity, Engraulis encrasicolus (Pecquerie et al., 2009). The general idea is that $E_{R}$ is partitioned into an unripe reproduction buffer $E_{R}^{0}$ and ripe batch material $E_{R}^{1}$ such that: $E_{R}=E_{R}^{0}+E_{R}^{1}$ (fig. $1 \mathrm{~B}$ ).

We implement the same rules published in Pecquerie et al. (2009, table 1), with three subtle differences:(a) batch preparation is triggered at time $t=t_{b}$, (b) batch preparation stops when energy is taken from $E_{R}^{0}$ to cover somatic maintenance and (c) batch energetic density $\left[E_{B}\right](\mathrm{J}$ $\mathrm{cm}^{-3}$ ) remains constant. Spawning can only happen when lights go on (unique event in a day) and when $E_{R}^{1} \geq\left[E_{B}\right] V$, with $\left[E_{B}\right] V$ the amount of energy in a spawned batch. 
Allocation to reproduction cumulates in $E_{R}^{0}$ (fig. 1B). If batch preparation is initiated ( $t>$ $t_{b}$ ) energy is mobilized from $E_{R}^{0}$ at maximum mobilization rate for an individual of size $L$ : $\dot{p}_{C m}$ (eq. (B.4), Online Appendix B). Energy is resorbed from the buffer to cover somatic maintenance if $\kappa \dot{p}_{C}<\dot{p}_{M}$ (see fig. 1) in which case batch preparation stops. We assumed that the reproduction overheads $\left(1-\kappa_{R}\right)$ are incurred during batch preparation. Dynamics of $E_{R}^{1}$ and $E_{R}^{0}$ are specified in Online Appendix B, table B.2. After a spawning event $t_{s}(\mathrm{~d})$ the amount of energy in the reproduction buffer is:

$$
E_{R}\left(t_{s}+d t\right)=E_{R}\left(t_{s}\right)-\left[E_{B}\right] V\left(t_{s}\right)
$$

The initial amount of energy in the egg $E_{0}(\mathrm{~J})$ is calculated using the maternal effect rule: reserve density $[E]=E / L\left(\mathrm{~J} \mathrm{~cm}^{-3}\right)$ of the mother equals $[E]$ of the offspring (Kooijman, 2009).

\subsection{Starvation}

As stated previously, if the flux of mobilized reserve is too small (i.e. $\kappa \dot{p}_{C}<\dot{p}_{M}$, fig. 1A), energy is first taken from $E_{R}^{0}$ to cover somatic maintenance. In this case specific growth rate $\dot{r}\left(\mathrm{~d}^{-1}\right)$ is 0 (table B.2, Online Appendix B). When there is no energy left in the reproduction buffer then $\dot{r}<0$ and energy is degraded from structure to cover $\dot{p}_{M}$ with efficiency $\kappa_{G}$ (table 1; Eq. (B.2), Online Appendix B).

Maturity maintenance $\dot{p}_{J}$ also needs to be paid, and in the event that reserve mobilization is insufficient for maturity maintenance, i.e. $(1-\kappa) \dot{p}_{C}<\dot{p}_{J}$, Augustine et al. (2011b) specified

how maturity level might decay exponentially with specific decay rate $\dot{k}_{J}^{\prime}\left(\mathrm{d}^{-1}\right)$. We set $\dot{k}_{J}^{\prime}$ to zero in this study, with the implication that no rejuvenation occurs. The penalty for not (or only partially) covering maturity maintenance comes theoretically with increased hazard $\left(\dot{h}_{J} \mathrm{~d}^{-1}\right)$ which would show up in the data as decreasing the survival probability because organisms become more susceptible to pathogens, bad water quality etc. We assume however that experimental conditions are favourable enough to health of individuals that such hazard is null.

\subsection{One compartment toxico-kinetic module}

We implement the simplest model formulation for specifying internal concentration: the one compartment toxico-kinetic module with time varying coefficients proposed by Kooijman and van Haren (1990); Kooijman (2010, Chap.6 pp.223-234) provides a more detailed discussion.

We make the simplified assumption that uptake is only from dissolved $\mathrm{U}$ in the water. In short, uptake is taken proportional to external concentration $c_{d}(\mathrm{nM})$. Elimination $\dot{k}_{e}\left(\mathrm{~d}^{-1}\right)$ is taken proportional to internal concentration $\left[M_{Q}\right]\left(\mathrm{nmol} \mathrm{cm}^{-3}\right)$ and inversely proportional to length (Kooijman, 2010; Jager and Zimmer, 2012).

Biomass is partitioned into $V, E$, and $E_{R}$ (table 2). Partitioning of $\mathrm{U}$ in the organism is assumed to be instantaneous between reserve material $\left(E+E_{R}\right)$ and structure $V$. Total mass of $\mathrm{U}$ in body, $M_{Q}(\mathrm{nmol})$ has contributions from mass of $\mathrm{U}$ in $V\left(M_{Q V}\right), E\left(M_{Q E}\right)$, and $E_{R}$ $\left(M_{Q R}\right)$. Let's consider $M_{E R}(\mathrm{~mol})$ as the total mass of reserve material $\left(M_{E R}=\left(E+E_{R}\right) / \bar{\mu}_{E}\right)$.

$$
M_{Q}=M_{Q V}\left(1+P_{E V} \frac{M_{E R}}{M_{V}}\right)=M_{Q V} P_{W V}
$$


with $M_{V}=V \frac{d_{V}}{w_{V}}$, mass of $V(\mathrm{~mol})$ and $w_{V}$ the molar weight of $V\left(\mathrm{~g} \mathrm{~mol}^{-1}\right)$. Kinetics of internal concentration amount to:

$$
\frac{d}{d t}\left[M_{Q}\right]=\frac{\dot{k}_{e}}{l}\left(P_{V d} c_{d}-\left[M_{Q}\right] P_{V W}\right)-\left[M_{Q}\right] \dot{r}
$$

with $P_{V d}\left(\mathrm{l} \mathrm{cm}^{-3}\right)$ the biomass / environmental uranium partition coefficient, $l$ a dimensionless scaled length measure $l=L / L_{m}\left(L_{m}=\kappa\left\{\dot{p}_{A m}\right\} / \dot{p}_{M}\right)$ and $c_{d}$ the environmental concentration of $\mathrm{U}(\mathrm{nM})$. Density of $\mathrm{U}$ in reserve material $m_{Q E R}$ is given by

$$
m_{Q E R}=\frac{M_{Q}-M_{Q V}}{M_{E R}}
$$

Total internalized U after a spawning event amounts to:

$$
M_{Q}\left(t_{S}+d t\right)=M_{Q}\left(t_{s}\right)-\frac{m_{Q E R}\left(t_{S}\right)}{\bar{\mu}_{E}}\left[E_{B}\right] V\left(t_{s}\right)
$$

\subsection{Characterizing effects on survival or on metabolism}

Survival probability $(S,-)$ is modelled as a sum of contributing hazards:

$$
\dot{h}=\dot{h}_{\mathrm{acc}}+\dot{h}_{\mathrm{a}}+\dot{h}_{\mathrm{J}}+\dot{h}_{\mathrm{Q}}+\dot{h}_{\mathrm{sh}}
$$

and

$$
\frac{d S}{d t}=-\dot{h} S
$$

Contributions from ageing $\dot{h}_{\mathrm{a}}$ and from rejuvenation $\dot{h}_{\mathrm{J}}$ are neglected. As stated in Augustine et al. (2011b), hazard from shrinking $\dot{h}_{\mathrm{sh}}$ is infinite with instantaneous death when a certain shrinking threshold is crossed. This case is also not addressed in this study. Contributions from accidents $\dot{h}_{\text {acc }}$ are estimated directly from control data (Bourrachot et al., 2008) and contributions from $\mathrm{U} \dot{h}_{\mathrm{Q}}$ are taken proportional to $\left[M_{Q}\right]$ such that:

$$
\dot{h}_{Q}=\dot{b}_{\dagger}\left(\left[M_{Q}\right]-\left[M_{Q 0}^{\mathrm{S}}\right]\right)_{+}
$$

where $\dot{b}_{\dagger}$ is the killing rate $\left(\mathrm{cm}^{3} \mathrm{nmol}^{-1} \mathrm{~d}^{-1}\right)$ and $\left[M_{Q 0}^{\mathrm{s}}\right]$ is the no effect internal concentration for survival.

Effects of uranium on measurable quantities such as growth and reproduction are assumed to occur when $\left[M_{Q}\right]>\left[M_{Q 0}^{*}\right]$, with * the metabolic process targeted by uranium. As we saw previously, there are 7 metabolic processes defined in the model (fig. 1A). An effect on a metabolic process is captured by the modification of the model parameter associated with that process (table 1$)$. In the neighbourhood of $\left[M_{Q 0}^{*}\right]$ for process ${ }^{*}$, the intensity of effect is taken linear to $\left[M_{Q}\right]$ by multiplying the targeted parameter with the dimensionless stress function $s$ :

$$
s=\frac{\left(\left[M_{Q}\right]-\left[M_{Q 0}^{*}\right]\right)_{+}}{\left[M_{Q T}^{*}\right]}
$$

with $\left[M_{Q T}^{*}\right]$ the tolerance concentration for process $*$. In short two extra parameters are required for modelling effects on metabolism and two extra parameters for modelling effects on survival. 


\section{$3 \quad$ Materials and Methods}

\subsection{Animal maintenance}

Adult fish were acquired from a commercial fish breeder (Elevage de la grande rivière, Lyon, France) and were almost a year old upon arrival. There were three experimental conditions. Each condition consisted of one holding tank containing 5 aquaria. The aquaria were permeable to holding tank water. There were 4 fish per aquaria (2 female and 2 male) and 20 fish (forming 10 couples) per experimental condition. Upon arrival fish were acclimated to the experimental system for $28 \mathrm{~d}$. During this time small groups in each aquaria were gradually thinned to 4 fish per aquaria (2 male and 2 female) in each of the 3 experimental conditions. Then followed $35 \mathrm{~d}$ acclimation to individual feeding and reproduction protocol which was identical to that described in Augustine et al. (2011a). Individuals were sometimes switched places to promote forming successful couples. After exposure, each fish remained in its position until the end of the experiment. Exposure duration was $37 \mathrm{~d}$. Fish were individually fed Ad libitum Tetramin $^{\mathrm{TM}}$ granules 2-3 times daily. Animals were kept in synthetic water. $\mathrm{T}=26.2^{\circ} \mathrm{C} \pm 0.7$, $26.3^{\circ} \mathrm{C} \pm 0.6$ and $26.4^{\circ} \mathrm{C} \pm 0.7$ (mean $\pm \mathrm{sd}$ ) in each respective condition over the entire acclimation and exposure duration; $\mathrm{pH}=6.5 \pm 0.1$ (mean \pm sd) in all three conditions over the entire acclimation and exposure duration; electrical conductivity was $191 \pm 8,192 \pm 8$ and $193 \pm 8 \mu \mathrm{S} \mathrm{cm}^{-1}$ (mean \pm sd) in each of the three respective conditions over the entire acclimation period and was not measured during exposure. $\mathrm{NO}_{2}^{-}$was monitored daily for the first $18 \mathrm{~d}$ of acclimation and was subsequently monitored about thrice weekly. $\mathrm{NO}_{2}^{-}$concentration, $0.08 \pm 0.05$ (mean \pm sd), never exceeded $0.2 \mathrm{mg} \mathrm{l}^{-1}$; this is acceptable with regards to zebrafish health (Lawrence, 2007). Additional details relevant to make up of the experimental system, $\mathrm{pH}$ and water composition, and feeding are provided in Online Appendix A.

\subsection{Experimental conditions}

Fish were exposed to three different concentrations of depleted uranium: 0, 84 and $420 \mathrm{nM}$. To compensate for daily absorption of $U$ (by experimental system, biofilms, algae or faeces) exposure media was continuously adjusted with uranium from a concentrated stock solution prepared in 1L glass bottles. The solution was released drop by drop into exposure media close to the internal recirculating pump (and away from individual aquaria) via capillaries and debit was regulated to approximately $0.04 \mathrm{l} \mathrm{h}^{-1}$. The stock solution was prepared on the onset of each day according to $U$ absorption by the experimental system assessed the previous day. Thus after a week the exposure concentration was stabilized to nominal concentration of 84 and $420 \mathrm{nM} \mathrm{U}$ (fig. A.1, Online Appendix A) in each respective condition.

\subsection{Water sample analysis}

Water samples were collected 2-3 times daily during the acclimation and exposure periods. Major anion concentrations were analysed by ionic chromatography (Dionex DX-120, Sunnyvale, CA, USA). Major cation and uranium concentrations were measured after $2 \%$ (v/v) $\mathrm{HNO}_{3}$ acidification by means of inductively coupled plasma-atomic emission spectrometry (ICP-AES; Optima 4300DV, Perkin-Elmer, Wellesley, MA, USA). Uranium detection limit is $42 \mathrm{nM}$. 


\subsection{Biometry}

Dorsal and lateral portraits of each individual were taken weekly (Nikon D3000) to assess visible signs of strain. Furthermore, each individual has a distinct pattern of stripes used to confirm individual identity of each female at the end of the experiment.

Reproduction was assessed by forming couples (1:1 male to female ratio) in the evening and counting the number of eggs spawned the following morning. The same couples were maintained during the $37 \mathrm{~d}$ exposure. Egg output $N$ of each individual was assessed on a daily basis.

At the end of the experiment fish were sacrificed, weighed and measured with a ruler from tip of to snout to base of caudal fin (standard length). Two females in the control condition were not weighed (females 3 and 4 in fig. 2). We provide estimates for their final wet mass as: $W_{w} \approx W_{1}\left(L_{f 1} / L_{f}\right)^{3}$, with $L_{f}$ observed final length of female 3 or 4 and $W_{1}$ and $L_{f 1}$ the observed wet mass and final length of female 1.

Final $\left\langle M_{Q}\right\rangle_{d}$ was determined for a random sub sample of 10 individuals in the 84 and 420 $\mathrm{nM}$ conditions in addition to 5 random control individuals.

After each spawn, eggs were rinsed in clean medium and placed in petri dishes and photographed (Nikon D3000) from above on a macro bench (VWR). Eggs were subsequently counted manually on photos (using Adobe ${ }^{\odot}$ Photoshop $^{\odot}$ CS4 11.0 and ImageJ 1.43u). Live and dead eggs were recorded.

3 samples of 10 live eggs were placed in 3 (pre-weighed) aluminium capsules (VWR $13 \mathrm{x}$ $5 \mathrm{~mm}$ width $\mathrm{x}$ height). Capsules were dried at $60^{\circ} \mathrm{C}$ for a minimum of $48 \mathrm{~h}$ then weighed on an ultra micro balance (Sartorius SE2, Göttingen, Germany). The samples were kept in an airtight plastic chamber over dessicated silica crystals after removal from $60^{\circ} \mathrm{C}$ oven and before weighing.

Adult fish were dried for $6 \mathrm{~d}$ at $60^{\circ} \mathrm{C}$. Whole body dry tissue was placed in a small glass beaker and digested in $8 \mathrm{ml}$ of $65 \% \mathrm{HNO}_{3}$ (Sigma Aldrich). Samples were evaporated to incipient dryness on a sand tray at $120-150^{\circ} \mathrm{C}$. The digestion process was followed by a $2 \mathrm{ml}$ $\mathrm{H}_{2} \mathrm{O}_{2}$ attack and evaporation to incipient dryness followed by a $2 \mathrm{ml} 65 \% \mathrm{HNO}_{3}$ digestion and evaporation to incipient dryness. These last two steps were repeated three times. Digested mineral residues were suspended in $5 \mathrm{ml}$ of $2 \% \mathrm{HNO}_{3}$ (v/v) acidified ultrapure water for ICPAES analyses.

\subsection{Other data and parameter estimation}

In addition to the $37 \mathrm{~d}$ exposure described above, data from 4 additional studies were also analysed with the DEB model. Barillet et al. (2005) and Barillet et al. (2011) present adult bioaccumulation data (nmol U per g whole body wet mass: $\left\langle M_{Q}\right\rangle_{w}$ ) at several exposure concentrations (table A.1, Online Appendix A). Bourrachot et al. (2008) demonstrate toxicological effects of $\mathrm{U}$ on embryo and early juvenile zebrafish growth and survival in combination with bioaccumulation. Bourrachot (2009) further demonstrates a decrease in total egg output $(N$, \# eggs) with increasing U concentrations (84 and $1054 \mathrm{nM}$ ) in combination with elimination of U through reproduction. Exposure conditions were similar across all experiments (table A.1, Online Appendix A).

DEB model parameters for zebrafish were already obtained in Augustine et al. (2011a) (table 1) using the covariation method (Lika et al., 2011a,b). We made two minor adjustments to 
parameter values: the specific density of structure $d_{V}=0.2$ instead of $0.15 \mathrm{~g} \mathrm{~cm}^{-3}$ and reserve's chemical potential $\bar{\mu}_{E}=5.0010^{5} \mathrm{~J} \mathrm{~mol}^{-1}$ instead of $5.5510^{5}$. We updated the parameter estimation files provided in Augustine et al. (2011a) with these values and the overall fit is slightly better with a growth conversion efficiency $\kappa_{G}=0.9$ (table 1) instead of 0.7. Primary energy parameters and parameters linking energy to volume or mass are herein treated as given. For simplicities sake, concentration of $\mathrm{U}$ in the water $c_{d}(t)$ is taken constant and equal to nominal exposure concentration for each experiment.

\subsubsection{Fitting the model to individual data for each female}

We perform the comparative analysis of data from Bourrachot (2009) and from our $37 \mathrm{~d}$ reproduction trial. The three exposure conditions in Bourrachot (2009) will hereafter be referred to as: $15 \mathrm{~d}-\mathrm{U} 0$ for controls $(\mathrm{n}=7), 15 \mathrm{~d}-\mathrm{U} 84$ for individuals exposed to $84 \mathrm{nM}(\mathrm{n}=8)$ and $15 \mathrm{~d}-$ U1054 for individuals exposed to $1054 \mathrm{nM}(\mathrm{n}=7)$. Each individual was first exposed to 0, 84 or $1054 \mathrm{nM} \mathrm{U}$ for $20 \mathrm{~d}$ and then placed in clean water for $15 \mathrm{~d}$. During exposure both sexes were separated. During depuration individuals were set up as triplets (1:2 female:male sex ratio), allowed to reproduce and daily $N$ was recorded for each individual female. Alternatively, the three exposure conditions in the reproduction trials presented in this study will hereafter be referred to as: $37 \mathrm{~d}-\mathrm{U} 0$ for controls $(\mathrm{n}=10)$, 37d-U84 for individuals exposed to $84 \mathrm{nM} \mathrm{U}(\mathrm{n}=10)$ and $37 \mathrm{~d}-\mathrm{U} 420$ for individuals exposed to $420 \mathrm{nM} \mathrm{U}(\mathrm{n}=10)$. The DEB model is fit to observed $N$ (sum of live and dead eggs) and final $W_{w}$ for each female in both experiments by adjusting $f$ and initial conditions. We also fit predicted $W_{d}^{0}$ to that observed for each individual female in the $37 \mathrm{~d}$ trial using the maternal effect rule. In the $15 \mathrm{~d}$ experiment we found that $t_{b}$ was a free parameter, while in the $37 \mathrm{~d}$ experiment we assumed that $t>t_{b}$ on the basis that couples were already formed during $35 \mathrm{~d}$ preceding exposure. For the $15 \mathrm{~d}$ trial we assumed that energy cumulated in $E_{R}$ during the first $20 \mathrm{~d}$ and spawning was only allowed to be triggered during depuration. Batch energetic density $\left[E_{B}\right]$ was found to vary between females and consequently

was estimated for each one. To remove a parameter we computed initial amount of structure as 0.94 the final amount of structure, assuming negligible growth during each experiments. Thus $L(0)=0.94 \delta_{M} L_{f}$. For comparison purposes between experiments we assumed $20 \%$ difference between standard and total length and expressed all lengths as total length (tip of snout to end of caudal fin). Toxicity parameters $P_{V d}, P_{E V}$ and $\dot{k}_{e}$ are estimated for all of the females in both experiments.

$W_{w}$ was computed for each female with and without the buffer handling rules. With buffer handling rules wet mass after spawning amounts to:

$$
W_{w}\left(t_{S}+d t\right)=W_{w}\left(t_{S}\right)-6 \frac{w_{E}}{\bar{\mu}_{E}}\left[E_{B}\right] V\left(t_{s}\right)
$$

and without the buffer handling rules, considering the observed amount of eggs spawned $N_{\text {obs: }}$ :

$$
W_{w}\left(t_{S}+d t\right)=W_{w}\left(t_{S}\right)-6 N_{\mathrm{obs}} W_{d}^{0}
$$

The computation of wet mass in Eq. (8) serves as a constraint for value of initial amount of reserve material for a given combination of $f$ and $L(0)$. Initial conditions, ingestion level and batch energetic density for each female in the $37 \mathrm{~d}$ and $15 \mathrm{~d}$ experiments can be found in table C.3, 
Online Appendix C. The observed lengths of each of the females from both experiments are plotted in fig. C.2, Online Appendix C. Finally, the estimated values of the toxicity parameters can be found in table C.4, Online Appendix C.

\subsubsection{5 day embryo and early juvenile experiments}

We assume $E_{0}=1.7 \mathrm{~J}$ (calculated with maternal effect rule taking $f=1$ for the mother). Bourrachot et al. (2008) presents data from 0 to $15 \mathrm{~d}$ and the authors started feeding early juveniles on day 9; control mortality also increases after $9 \mathrm{~d}$. The increase in control mortality in combination with low control $W_{d}$ indicate that food is hardly eaten and suggests a water quality problem. For this reason we restrict analysis to the first $9 \mathrm{~d}$ where no extra organic material (liquid food) was added to the system. Nonetheless, $f$ is freely estimated after birth; the slight increase in mean control $W_{d}$ suggest some assimilation took place. $\delta_{M}$ was shown to increase during the early juvenile period with ontogeny of caudal fin (Augustine et al., 2011a), but such 'details' are here neglected. We calibrate an overall embryo $\delta_{M}$ to $L_{f}$ at $9 \mathrm{~d}$ (table 1 ).

\subsection{3 $20 \mathrm{~d}$ adult accumulation experiments}

We calibrated initial conditions to initial observed mean $W_{w}$ in each condition in Barillet et al. (2005) and to initial observed mean $W_{w}$ and $L_{f}$ in Barillet et al. (2011). Consequently, there are three free parameters for each condition in Barillet et al. (2005): $f, E_{R}^{0}(0)$ and $L(0)$ and only two free parameters for the condition in Barillet et al. (2011): $f$ and $E_{R}^{0}(0)$. Nominal $c_{d}$ for each condition in both studies are provided in table A.1, Online Appendix A. At each sampling time 10 random individuals were sampled and biometry and $\left\langle M_{Q}\right\rangle_{w}$ was recorded. In the study by Barillet et al. (2005) fish of both sexes were sampled, while in the study by Barillet et al. (2011) only males were sampled. We consider that males and females both possess the same model parameters (table 1).

\subsubsection{Estimation procedures}

The general strategy was to first fit the control model to all of the data sets to first determine that controls were correctly described and second confirm that the control model could not adequately describe the toxicity data. The second phase was to determine toxico-kinetic parameters and test which metabolic process $(*)$ was most likely impacted. We performed a sensitivity analysis (not shown) on the control model to ascertain what combination of effects on measurable quantities are obtained through which parameter modification. Thus we qualitatively assessed the three most likely metabolic processes $(*)$ targeted by uranium: $(\mathrm{G})$ uranium may be increasing cost of synthesis of a unit of structure: $\left[E_{G}^{s}\right]=\left[E_{G}\right](1+s),(\mathrm{M})$ uranium might be increasing volume-linked somatic maintenance $\left[\dot{p}_{M}^{s}\right]=\left[\dot{p}_{M}\right](1+s)$ and $(\mathrm{A})$ uranium may be decreasing surface-area linked assimilation $\left\{\dot{p}_{A m}^{s}\right\}=\left\{\dot{p}_{A m}\right\}(1-s)$. We fit the model three times to toxicity data; first assuming mode of action $G$, then mode of action $M$ and finally mode of action $A$. All computations were performed with Matlab (R2010b, Mathworks $\left.{ }^{\odot}\right)$. Parameters for modelling survival were estimated using the regression method (Kooijman, 1981). The simplex (Nelder-Mead) method was used to simultaneously minimise the weighted sum of squared deviations between model predictions and observations for all of the other data sets 
(Lika et al., 2011a). Parameter estimates were performed using freely downloadable software DEBtool (http://www.bio.vu.nl/thb/deb/).

\section{Results}

A rapid synthesis of the five studies teaches that effects are (somehow) related to internalized $\mathrm{U}$ and two different types of organism level effects were observed when comparing the early life stages to adults: an effect on growth and survival for the first and an effect on reproduction attributed to modified behaviour for the latter. Parameters provided in Augustine et al. (2011a) are in line with all of the control data of the 5 studies when (i) taking differences in feeding into account and (ii) using appropriate initial conditions.

Bourrachot (2009) concluded that the decrease in $N$ stemmed from a reduction in number of spawning events per female and suggested that this may be a consequence of $U$ induced impairment of sensory organs. Ovulation is in fact stimulated in female zebrafish though pheromones liberated by the partner and may be further influenced through female dominance structures, mate preference and spawning substrate (Gerlach, 2006; Spence et al., 2008). The purpose of our $37 \mathrm{~d}$ exposure experiment where daily $N$ was measured was to test this particular hypothesis. Surprisingly, we obtained conflicting results with the previous reproduction experiment. Whereas $20 \mathrm{~d}$ accumulation (without reproduction) to $84 \mathrm{nM}$ followed by $15 \mathrm{~d}$ depuration with reproduction showed strong impacts on mean cumulated $N$ (Bourrachot, 2009), our $37 \mathrm{~d}$ accumulation to $84 \mathrm{nM} \mathrm{U}$ with reproduction did not. However, the same decrease in $N$ stemming from drastic reduction in inter-spawning events was observed at $420 \mathrm{nM} \mathrm{U}$ as for the $1054 \mathrm{nM} \mathrm{U}$ condition in Bourrachot (2009). Absence of effects on adult growth and inexplicable variability in bioaccumulation in all of the experiments were also observed.

In the following sections we present results of fitting the control model to controls from both reproduction trials $(15 \mathrm{~d}$ and $37 \mathrm{~d}$ ). We then present results of fitting the control model to exposed females from both trials. We continue by explaining how effects on exposed females are captured by the perturbation of a specific physiological process. The last two sections compare model predictions with observations of adult whole body residues and embryo and early juvenile growth, bioaccumulation and survival.

\subsection{Control Reproduction trials}

At first, we assumed that even if some energy is taken from $E_{R}$ to cover somatic maintenance, batch preparation continues until $E_{R}$ is emptied. This does not fit the observations from both reproduction trials. The control data empirically support that batch preparation stops when not enough energy is mobilized from reserve to cover somatic maintenance. Predicted cumulated number of eggs and final wet mass for control females are presented in fig. 2. We plot predicted wet mass with and without buffer handling rules (Eq. (7) and (8)). We consider that fits are good when absence or presence of spawning is accurately depicted and predicted values of eggs per spawn is close to that observed. Predicted cumulated number of eggs is in line with observations for females 1,3, 4, 5, 8, 9 and 10 respectively. The spawning behaviour of females 2, 6 and 7 seems to escape buffer handling rules, but predicted final mass without buffer rules is in line with that observed. The mean of each prediction for cumulated number of 


\section{7d-U0}
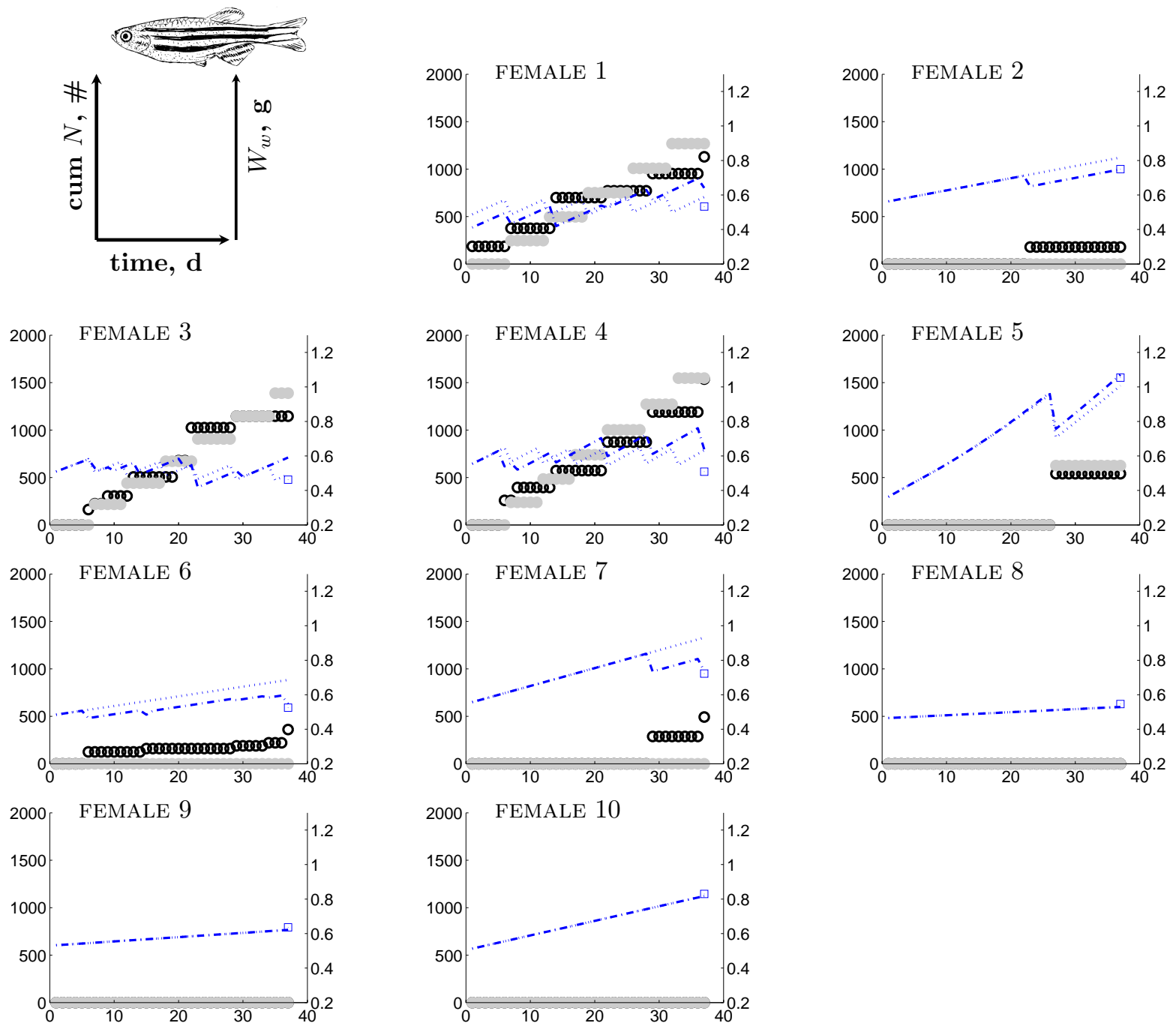

Figure 2: DEB Model predictions and observations for each control females in the 37d reproduction trial. Left y-axis: empty circles (black) represent observed cumulated (cum) number $N$ of eggs spawned (\#) and full circles (grey) represent model predictions. Right y-axis: dotted (blue) line: predicted wet mass $W_{w}(\mathrm{mg})$ computed with buffer handling rules, Eq. (7). Dot-dashed (blue) line: predicted $W_{w}$ computed without buffer handling rules, Eq. (8). Square symbol (blue): final observed $W_{w}$. Final observed $W_{w}$ was not measured for females 3 and 4 . We provide estimates for their final wet mass as: $W_{w} \approx W_{1}\left(L_{f 1} / L_{f}\right)^{3}$, with $L_{f}$ observe final length of female 3 (or 4 ) and $W_{1}$ and $L_{f 1}$ the observed final wet mass and final length of female 1. Initial conditions, batch energetic density and ingestion level for each female are recorded in table C.3, Online Appendix C. 
eggs spawned is compared with the mean of that observed for 15d-U0, 15d-U84 and 15d-U1054 (fig. 3A). Final predicted wet mass is compared with that observed for all three conditions (fig. 3B). Predictions for each replicate in all three conditions can be found in fig. E.6-E.8, Online Appendix E. $f,\left[E_{B}\right]$ and initial $E_{R}$ were found to differ between females (table C.3, Online Appendix C). $\left[E_{B}\right]$ seemed to be in the range of $1000-3000 \mathrm{~J} \mathrm{~cm}^{-3}$ with some exceptions (e.g. female 5 in $37 \mathrm{~d}-\mathrm{U} 0$ ). All of the control females spawned in $15 \mathrm{~d}-\mathrm{U} 0$, whereas 3 did not in 37d-U0. Furthermore females 2, 6 and 7 (37d-U0) kept most of the cumulated energy invested in reproduction inside the body. Total wet mass is partitioned into three types of material: $E$, $E_{R}$ and $V$ (table 2). The observed length gives an estimate for mass of $V$, but the assumption of constant food and an estimate of ingestion level $f$ are needed to quantify relative contributions of $E$ and $E_{R}$ to total biomass. Without observed initial $W_{w}$ the values of $f$ and $E_{R}(0)$ are not accurately fixed, especially if there is no spawning, since there is no handle on $f$. In this case several local minimums may exist in the parameter space comprising initial conditions and $\left[E_{B}\right]$. However, by computing $W_{w}$ without buffer handling rules we introduce a constraint on the lower boundary of initial $E_{R}$ for a given value of $f$. In the event the individual spawned, then $f$ also conditions the slope of cumulated $N$ in the $37 \mathrm{~d}$ trial. On the contrary, $f$ hardly conditions the slope of $N$ in the $15 \mathrm{~d}$ trial. Indeed, the slope of cumulated $N$ in the $15 \mathrm{~d}$ trial is conditioned by how much energy is in $E_{R}$ at the onset of the trial and more specifically how much of that energy is ripe batch material.

At first, we assumed $t>t_{b}$ at the onset of both reproduction trials, on the basis that the presence of a male stimulates ovulation in females. This assumption does not hold for the $15 \mathrm{~d}$ trial. Actually, considering a batch energy density of about 1000-2000 $\mathrm{J} \mathrm{cm}^{-1}, 5$ females out of 7 must already have had ripe batch material at the onset of the $15 \mathrm{~d}$ trial (table C.3, Online Appendix C). Predicted mean dry mass of an egg in each spawn, for each female in 37d-U0, are compared with the observed values (fig. 4). The results cannot prove that the maternal effect is accurate, but do strongly support linking zebrafish egg mass to maternal condition quantified by $[E]$. Assuming the maternal effect, then $[E]$ of female 4 seems to be underestimated. We estimated an $f$ of 0.94 for female 5 , but observed $W_{d}^{0}$ was still higher than predicted.

Three (37d trial) or four parameters (15d trial) are needed to specify initial conditions and buffer handling for a female. This makes one parameter per curve for the $37 \mathrm{~d}$ trial considering the three types of observations $N, W_{w}$ and $W_{d}^{0}$ and 2 parameters per curve for the $15 \mathrm{~d}$ trial considering the two observations $N$ and $W_{w}$. Lastly, predicted reproductive output in the $37 \mathrm{~d}$ experiment is less sensitive to initial value of $E_{R}$ than the $15 \mathrm{~d}$ trial. In the latter case the entire curve is conditioned by the amount ripe batch material available the first day of the trial. Females in the $37 \mathrm{~d}$ trial were on average longer than females in the $15 \mathrm{~d}$ trial (fig. C.2, Online Appendix C). The implication is that females in the $37 \mathrm{~d}$ trial have on average higher $V$ than in the $15 \mathrm{~d}$ trial. The DEB model takes somatic maintenance proportional to $V$, so the average minimum ingestion level for which somatic maintenance is covered is higher in the $37 \mathrm{~d}$ trial relative to the $15 \mathrm{~d}$ trial. We assumed a constant $\left[E_{B}\right]$ for each female, but in practice $\left[E_{B}\right]$ might be variable. Both reproduction trials sported very different protocols for evaluating reproductive output. The $15 \mathrm{~d}$ trial maintained both sexes in separate tanks for $20 \mathrm{~d}$ before placing each individual female with two male partners in small spawning chambers and in the $37 \mathrm{~d}$ trial couples were already formed 35d before the onset of the experiment. By incorporating experiment design, the model captures the pattern where cumulated number of eggs spawned 

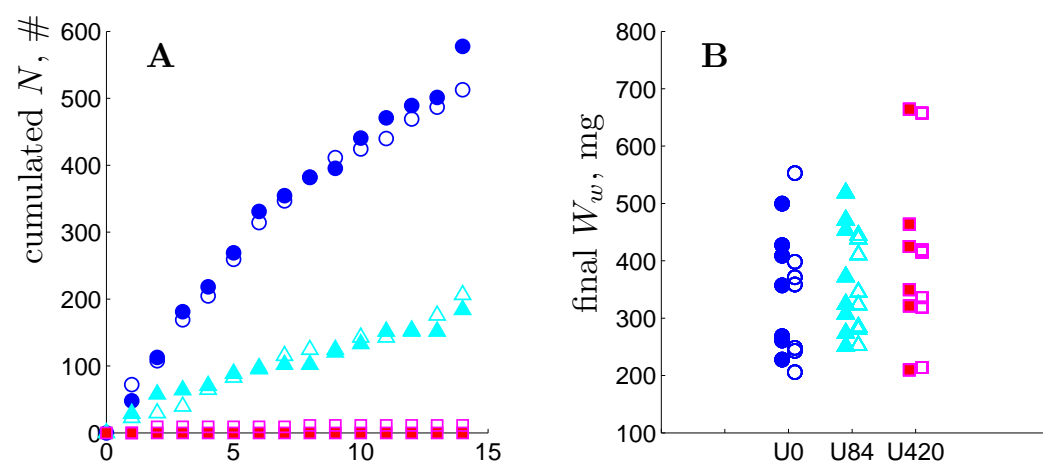

time, $d$
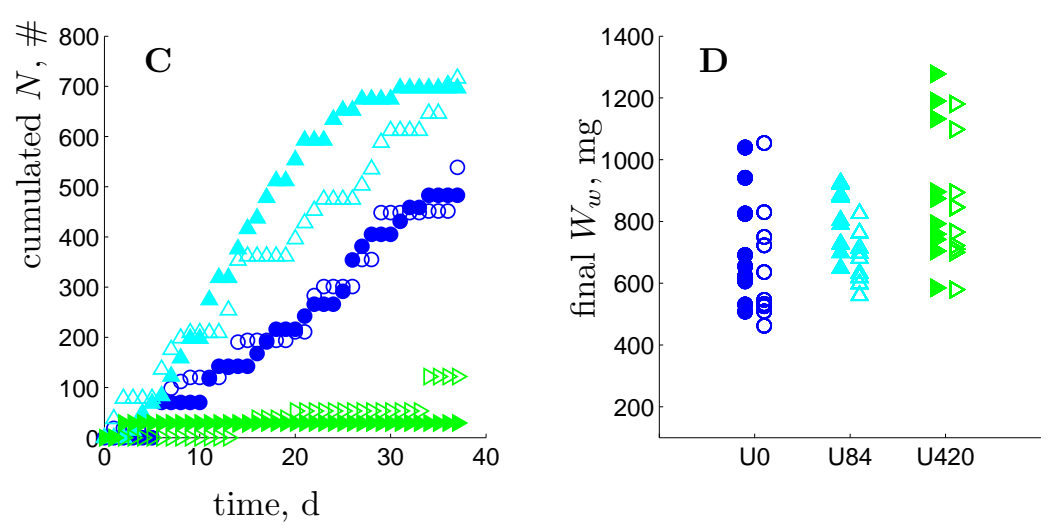

Figure 3: Full symbols: model predictions; empty symbols: observations; circles: controls; upwards triangles: exposed to $84 \mathrm{nM}$; sideways triangles: exposed to $420 \mathrm{nM}$; squares: exposed to $1054 \mathrm{nM}$. (A) mean cumulated number of eggs $(N)$ spawned for 15d trial. (B) Final observed and predicted wet mass $W_{w}$, mg for each individual in the $15 \mathrm{~d}$ trial. Model fits for each individual in the 15d trial can be found in Online Appendix E. (C) mean cumulated $N$ spawned during the $37 \mathrm{~d}$ trial. (D) Final observed and predicted wet mass $W_{w}, \mathrm{mg}$ for each individual in the $37 \mathrm{~d}$ trial. Model fits for each individual in the $37 \mathrm{~d}$ trial can be found in fig. 2, 5 and 6. 
rises steeply then saturates in the $15 \mathrm{~d}$ trial (fig. 3A) and alternatively where it rises with an approximately constant slope for females in the $37 \mathrm{~d}$ trial (fig. $3 \mathrm{C}$ ).

\subsection{Fitting the control model to exposed females}

The control model was fit to each exposed female in order to detect if the model for baseline metabolism could explain the observations simply on the basis of physiological differences in initial $E_{R}$ and/or feeding. The result is, certain exposed females indeed present quantitative energetic differences from predictions for baseline metabolism. There is a discrepancy between model predictions for cumulative reproductive investment relative to actual (indirectly) observed cumulative reproductive investment. This was clearly the case for three females in the 15d-U84 condition (not shown) and in all but one reproducing female in the 37d-U84 condition (fig. D.3, Online Appendix D). Incidentally, degrees of freedom for parameter estimation are higher in the $15 \mathrm{~d}$ experiment than in the $37 \mathrm{~d}$ experiment.

Initial slope of cumulated number of eggs spawned for females 2, 3, 6, 7 and 10 in 37d-U84 are suggestive of an $f$ of at least 0.8 (see estimated values in table D.5, Online Appendix D). The observed dry mass of an egg for females 2,3, 6 and 7 (fig. 4) are also supportive of a high $[E]$. When fitting the control model to $37 \mathrm{~d}-\mathrm{U} 84$ data we found that the wet mass computed with buffer handling rules Eq. (7) and predicted cumulated number of eggs spawned matched observations for the above mentioned females (fig. D.3, Online Appendix D). Predictions deviate from observations around $20 \mathrm{~d}$ for e.g. females 2, 3, 6 and 7 as does the wet mass computed without the buffer handling rules Eq. (8). This illustrates that less material is actually in the reproduction buffer than predicted for these females. The control model predictions are in harmony with observations for female 10 . The absence of spawning in the $37 \mathrm{~d}-\mathrm{U} 420$ and $15 \mathrm{~d}-$ U1054 conditions could be explained by a reduced $f$ in most instances (not shown). The $37 \mathrm{~d}-$ U84 data sets provided the most convincing evidence of uranium-induced metabolic deviations, because of the varying degrees of deviations between real and predicted wet mass and cumulated number of eggs spawned for a large number of females.

\subsection{Mode of action of uranium on females in reproduction trials}

The three modes of action $(\mathrm{G}),(\mathrm{M})$ and $(\mathrm{A})$ were tested to determine which one best captures uranium induced effects on female cumulated reproductive investment at low concentrations. As stated before, growth is negligible for females in the $37 \mathrm{~d}$ trial and most likely negligible

for a number of females in the $15 \mathrm{~d}$ trial. We simulated effects of increasing $\left[E_{G}\right]$ considering experimental conditions of both reproduction trials and found that cumulated reproductive investment would hardly be impacted considering individuals of the size used and experiment duration (fig. D.5, Online Appendix D). Thus, mode of action (G) cannot explain the observations.

Since, decreasing $f$ seemed to increase goodness of fit for all exposure conditions (when using only the control model), then mode of action (A) is a likely candidate to explain effects. Ingestion level can be high, but by decreasing surface area-linked assimilation it is possible to emulate a decline in the slope of reproductive output due to reduced reserve mobilization. Finally, energy assimilated in reserve would no longer suffice to cover somatic maintenance 

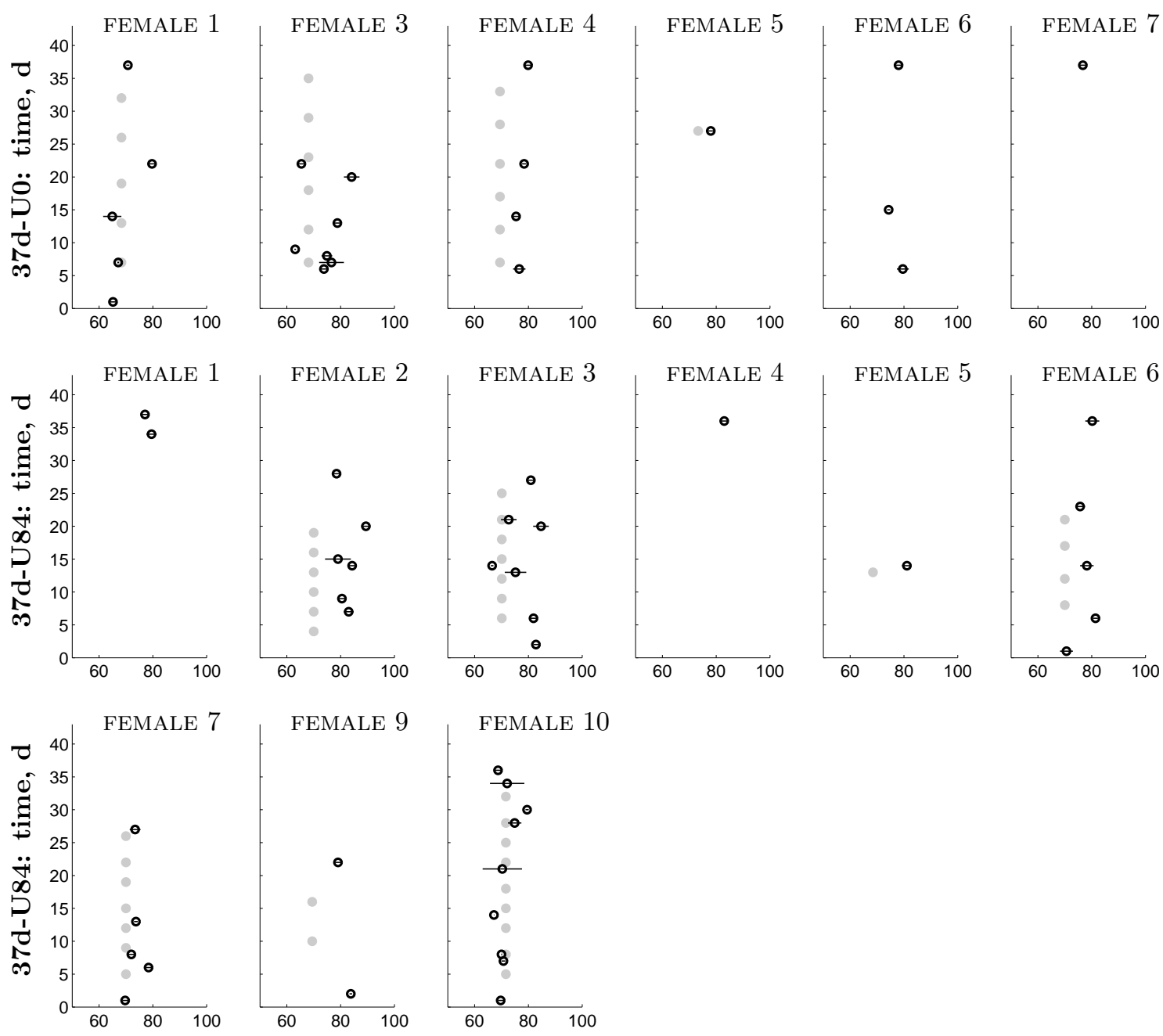

FEMALE 10
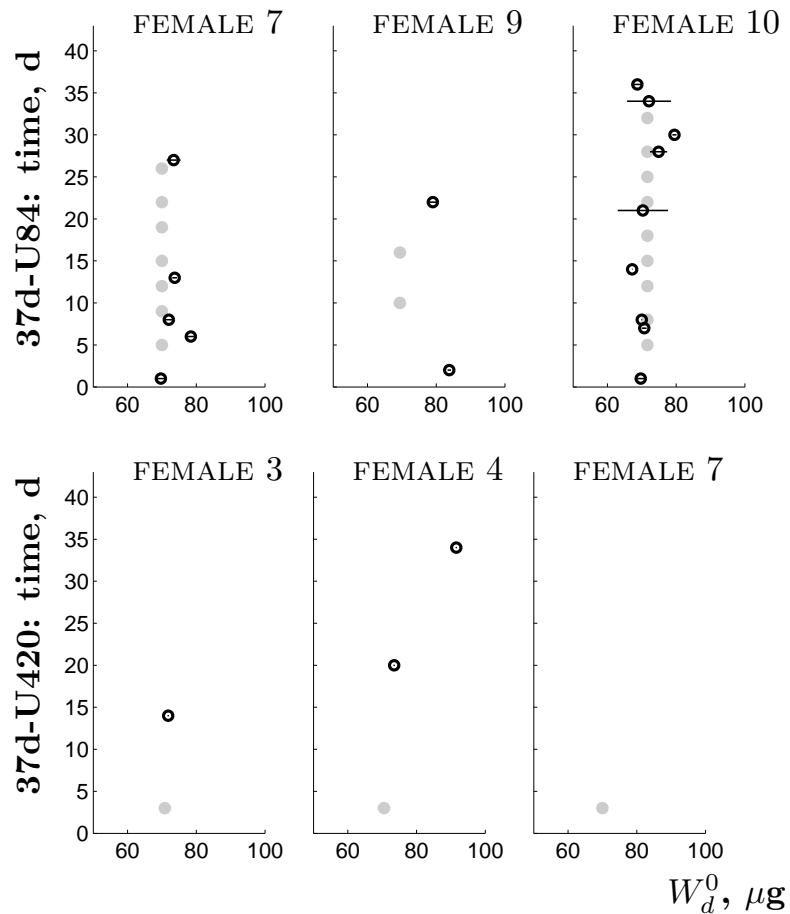

Figure 4: $\quad$ Egg dry mass $W_{d}^{0}(\mu \mathrm{g})$ of a live egg for each spawn of various females in the $37 \mathrm{~d}$ reproduction trial. First row: controls; second and third row: U-84; fourth row: U-420. Empty (black) circles: observed mean $W_{d}^{0}$; bars: standard deviation $(\mathrm{n}=3$, see text for protocol). Full (grey) circles: predicted $W_{d}^{0}$ considering the maternal effect rule. Predicted $W_{d}^{0}$ is constant because $f$ is constant and thus $[E]$ is constant (weak homeostasis, Kooijman, 2010, Chap.2). 


\section{$37 \mathrm{~d}-\mathrm{U} 84$}
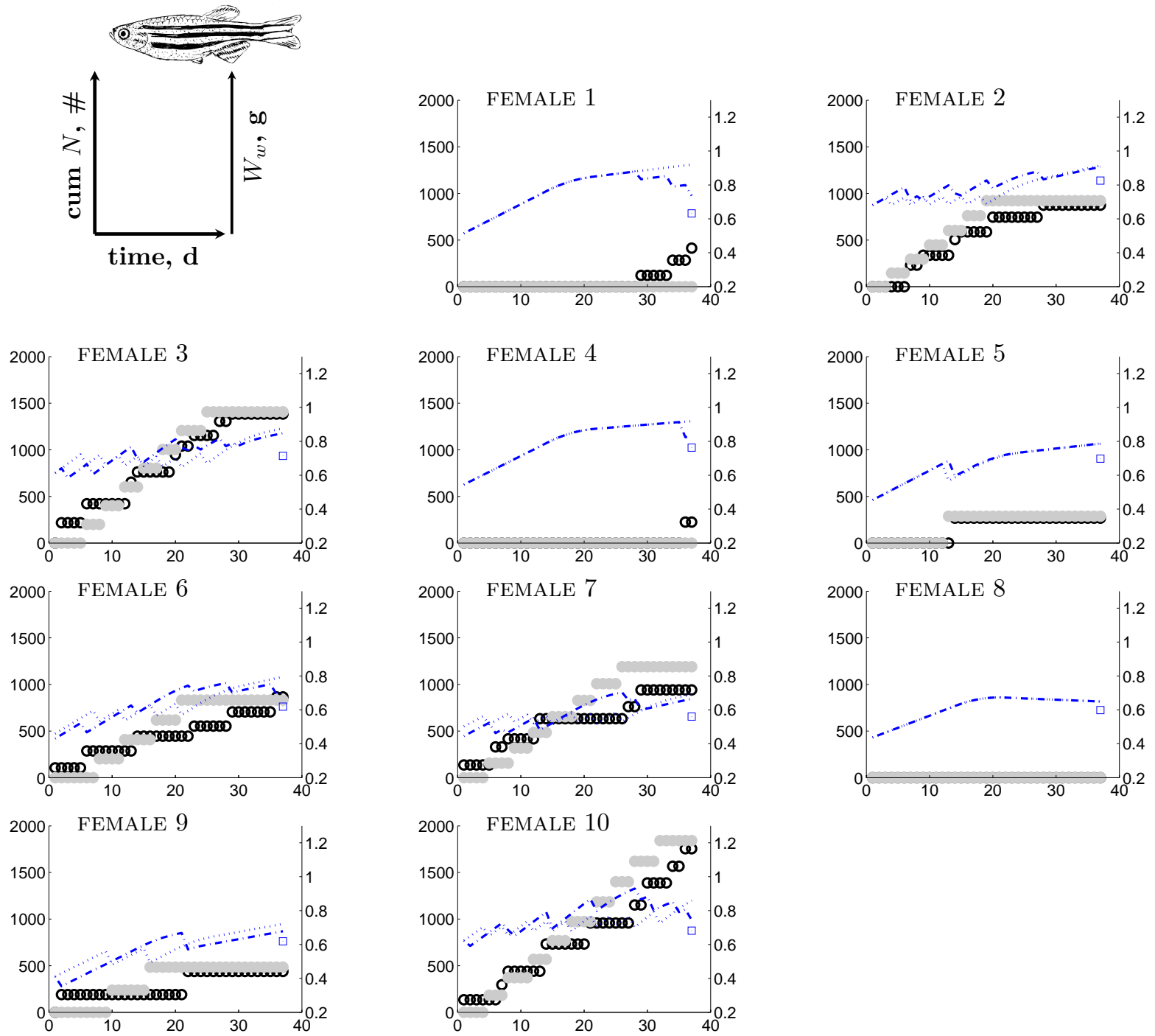

Figure 5: DEB Model predictions and observations for each female exposed to $84 \mathrm{nM}$ for $37 \mathrm{~d}$ assuming that uranium increases volume-linked somatic maintenance. Left y-axis: empty circles (black) represent observed cumulated (cum) number $N$ of eggs spawned (\#) and full circles (grey) represent model predictions. Right y-axis: dotted (blue) line: predicted wet mass $W_{w}(\mathrm{mg})$ computed with buffer handling rules, Eq. (7). Dot-dashed (blue) line: predicted $W_{w}$ computed without buffer handling rules, Eq. (8). Square symbol (blue): final observed $W_{w}$. Initial conditions, batch energetic density and ingestion level for each female are recorded in table C.3, Online Appendix C. 


\section{$37 \mathrm{~d}-\mathrm{U} 420$}
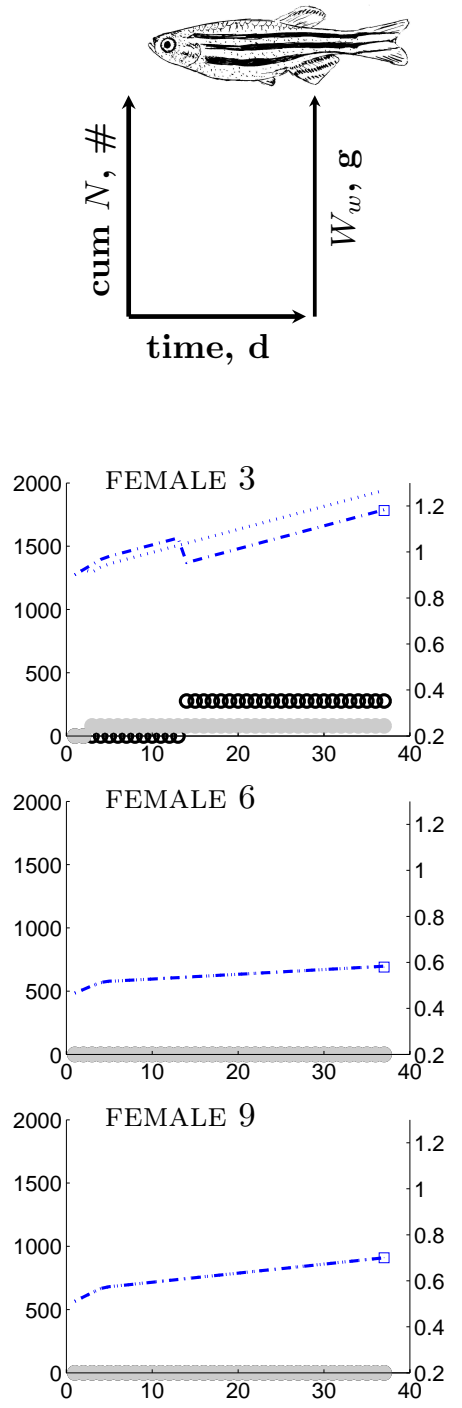
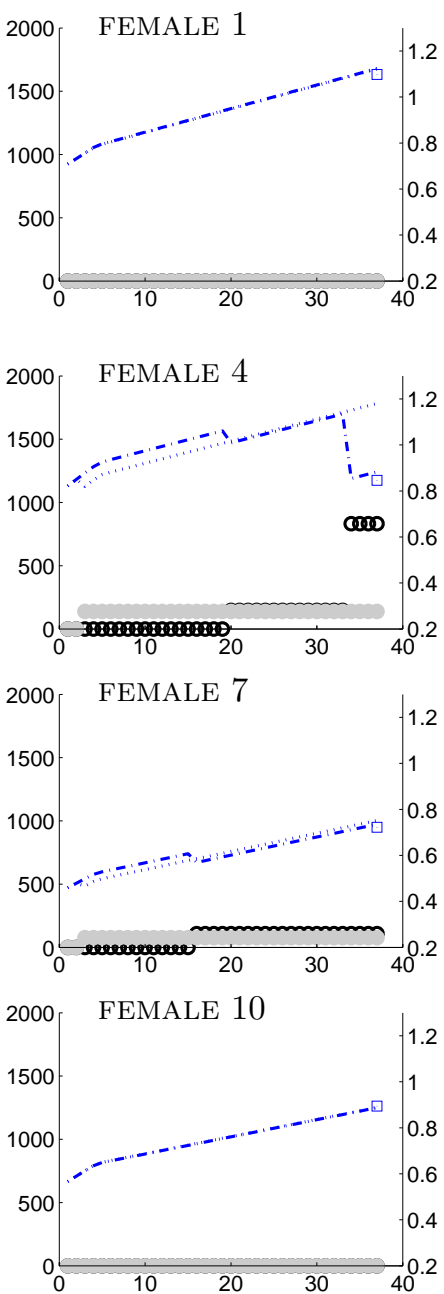
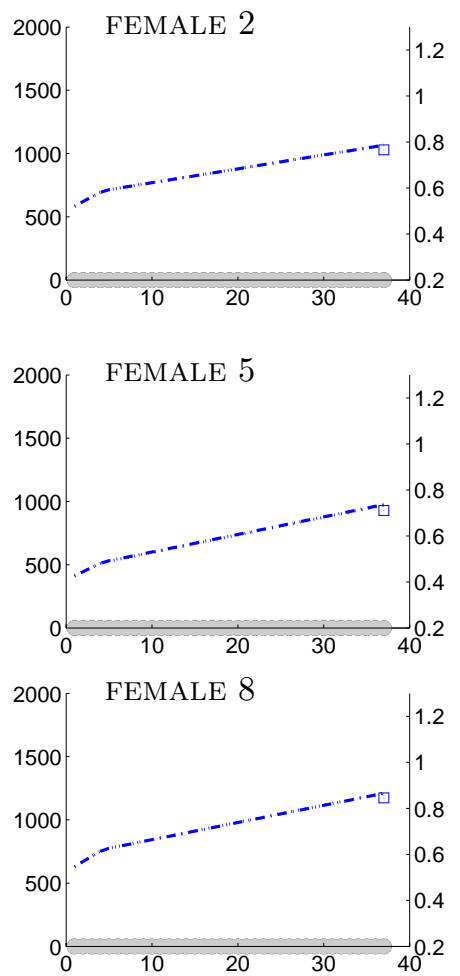

Figure 6: DEB Model predictions and observations for each female exposed to $420 \mathrm{nM}$ for $37 \mathrm{~d}$ assuming that uranium increases volume-linked somatic maintenance.. Left y-axis: empty circles (black) represent observed cumulated (cum) number $N$ of eggs spawned (\#) and full circles (grey) represent model predictions. Right y-axis: dotted (blue) line: predicted wet mass $W_{w}(\mathrm{mg})$ computed with buffer handling rules, Eq. (7). Dot-dashed (blue) line: predicted $W_{w}$ computed without buffer handling rules, Eq. (8). Square symbol (blue): final observed $W_{w}$. Initial conditions, batch energetic density and ingestion level for each female are recorded in table C.3, Online Appendix C. 
and reproduction would be inhibited. We fit the model considering mode of action (A) to the various females. In doing so, the decrease in $N$ was captured but final predicted $W_{w}$ still overshot observed $W_{w}$ for the females in the 37d-U84 condition. Mode of action (M) assumes an increase in volume-linked somatic maintenance costs. In this scenario, ingestion level can remain high as does reserve mobilisation. Spawning is brusquely inhibited when somatic maintenance is so high that $\kappa \dot{p}_{C}$ no longer suffices to pay it and energy is resorbed from the reproduction buffer. Since the amount of metabolic work associated with somatic maintenance is so high, a large amount of reproduction buffer material is burned to cover it. Mode of action (M) most satisfactorily captured final mass and observed cumulated $N$ for females in the 37d-U84 condition (fig. 5).

In the absence of further information we assumed mode of action (M). Individual model predictions for cumulated number of eggs spawned and final wet mass for $37 \mathrm{~d}-\mathrm{U} 84$ and $37 \mathrm{~d}-$ U420 are presented in fig. 5-6. The mean of all the model predictions for the $15 \mathrm{~d}$ trial are compared to mean observed cumulated number of eggs spawned in fig. 3A. We further plot mean observed cumulated number of eggs against the mean of the predictions for $37 \mathrm{~d}-\mathrm{U} 0$, 37d-U84 and 37d-U420 respectively (fig. 3C). The individual model fits (fig. 5) look better graphically than the mean of the predictions for 37d-U84 because the model assumes batch preparation stops immediately when energy is resorbed from $E_{R}$ to cover $\left[\dot{p}_{M}\right] V$ and that $\left[E_{B}\right]$ remains constant. If we had plotted observed mean cumulated number of eggs spawned with say standard deviations, the plot would be unreadable. We displayed both representations: individual females and means to illustrate that we are not comparing number of eggs produced, but indirectly quantifying cumulative energy investment in reproduction for each individual female. The latter depends on surface area (length) and feeding $(f)$ and is much less variable between females than spawning behaviour.

Finally, we draw attention to the higher mean reproductive output in the 37d-U84 condition relative to controls (fig. 3C). This is explained by the higher ingestion levels in the 37d-U84 (mean $f=0.79 \pm 0.05 \mathrm{sd}$ ) condition relative to controls (mean $f=0.68 \pm 0.13 \mathrm{sd}$ ). The mean ingestion level in $37 \mathrm{~d}-\mathrm{U} 420$ was $0.81 \pm 0.04 \mathrm{sd}$. The value of the partition coefficient $P_{E V}$ in combination with $\left[E_{B}\right]$ regulates how much and at what frequency $\mathrm{U}$ is eliminated through reproduction. As such, both parameters modulate dynamics of $\left[M_{Q}\right]$ relative to spawning behaviour. Within this modelling framework, spawning patterns manifested by the 10 females in 37d-U84 are captured by different kinetics of effects with female 8 and female 10 at two extremities of the effect spectrum. The more the female spawns, the more she eliminates and so the longer she stays beneath the no effect internal concentration. Female 10 for example spawned regularly all the way to the end while females 1,4 and 8 did not. In the first three cases the longer the inter-spawning interval the more intense is the increase in somatic maintenance. Females 1 and 4 did spawn towards the end of exposure, hampering firm conclusions.

Predictions using the control model match model predictions considering mode of action (M) for female 10 for (a) certain value(s) of $P_{E V}$. This is the reason why the simultaneous analysis of all the females in the 37d-U84 condition also allows us to fix the value of $P_{E V}$ for a certain combination of $\left[M_{Q 0}^{*}\right],\left[M_{Q T}^{*}\right],\left[E_{B}\right]$. The values of toxicity parameters used to obtain the fits provided for each of the exposed females in this study can be found in table C.4, Online Appendix C. The broad patterns in toxicity were captured assuming a no internal effect concentration for $\left[\dot{p}_{M}\right]\left[M_{Q 0}^{\mathrm{M}}\right]$ of $32 \mathrm{nmol} \mathrm{cm}{ }^{-3}$ and a tolerance concentration $\left[M_{Q T}^{\mathrm{M}}\right]$ of 28.9 
nmol cm$~^{-3}$. $P_{E V}$ is $13.1 \mathrm{~mol} \mathrm{~mol}^{-1}$. The biomass/ water partition coefficient for uranium was determined to be $19.1 \mathrm{l} \mathrm{cm}^{-3}$ and elimination rate $\dot{k}_{e}$ to be $0.003 \mathrm{~d}^{-1}$. This entails that the No Effect Concentration (NEC) of uranium in the exposure media is $\left[M_{Q 0}^{\mathrm{M}}\right] / P_{V d} \approx 1.7 \mathrm{nmol}$ $\mathrm{l}^{-1}$. If $\left[\dot{p}_{M}\right]$ continues to increase linearly to internal concentration even beyond $\left[M_{Q 0}^{\mathrm{M}}\right]$, then the individuals in the $37 \mathrm{~d}-\mathrm{U} 420$ and $15 \mathrm{~d}-1054$ as well as in the higher exposure conditions by Barillet et al. $(2005,2011)$ would have shrunk to death during the experiment. This is manifestly not the case. Portraits of all of the individuals at the end of the experiment (not shown) corroborate this. In the absence of further information (and to avoid numerical errors) we set a maximum value to $\left[\dot{p}_{M}\right]$ equal to $1000 \mathrm{~J} \mathrm{~cm}^{-3} \mathrm{~d}^{-1}$.

\subsection{Adult whole body residues}

Of the 10 random individuals sampled for measuring final $\left\langle M_{Q}\right\rangle_{d}$ in each condition of the $37 \mathrm{~d}$ reproduction trial, only four were females. Predicted $\left\langle M_{Q}\right\rangle_{d}$ are compared to that observed for four individual females in conditions 37d-U84 and 37d-U420 (fig. 7). Bourrachot (2009) presents

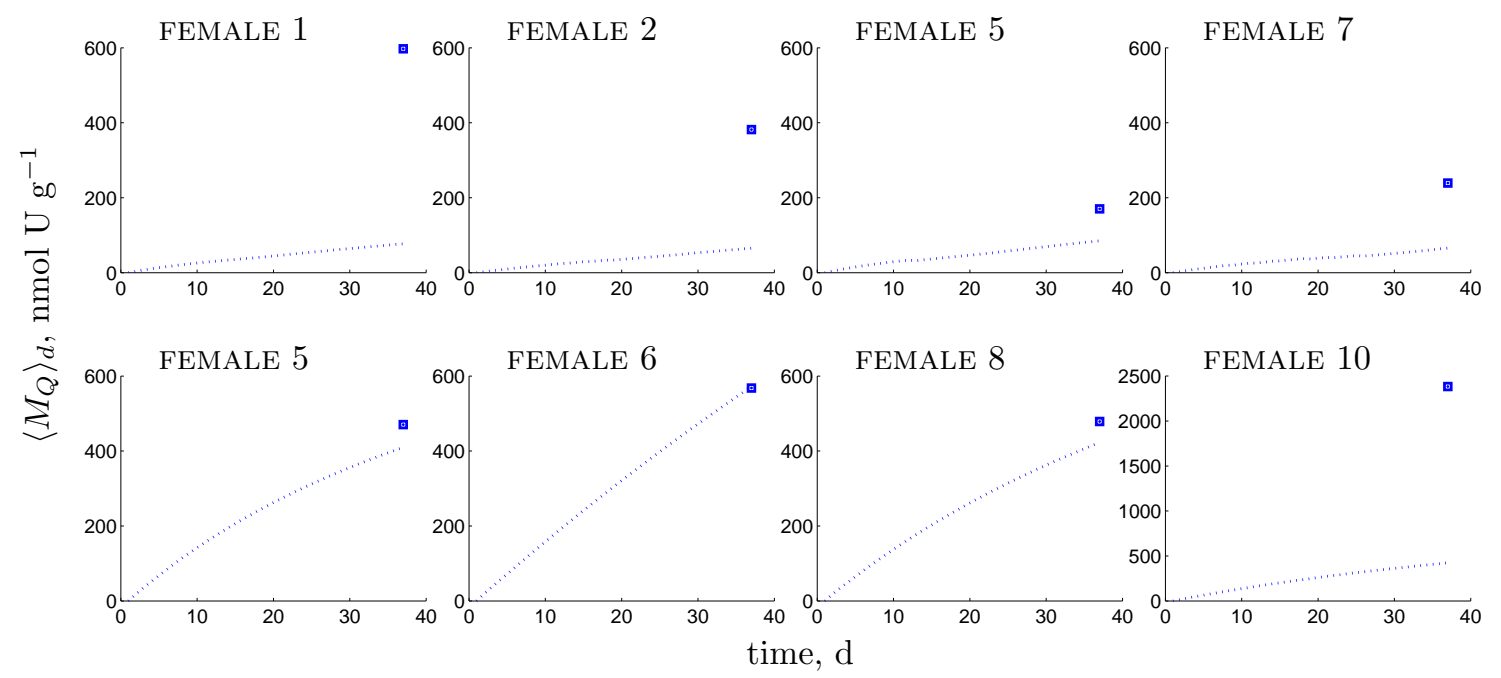

Figure 7: First row: U-87; second row: U-420. Square: final measured internal concentration $\left\langle M_{Q}\right\rangle_{d}$ (nmol g ${ }^{-1}$ dry mass); dotted line: predicted $\left\langle M_{Q}\right\rangle_{d}$.

mean $\left\langle M_{Q}\right\rangle_{d}$ at onset and end of the $15 \mathrm{~d}$ trial. There is some uncertainty in the interpretation of those $\left\langle M_{Q}\right\rangle_{d}$ measurements since the data are presented as mean concentrations in gonad and whole body excluding gonad respectively. The two concentrations cannot be added in a meaningful way to obtain whole body residues. Bourrachot (2009) also measured $\left\langle M_{Q}\right\rangle_{d}$ in the eggs of several females at the onset and the end of the $15 \mathrm{~d}$ trial in both exposure conditions. The author found mean values of 540 and $35 \mathrm{nmol} U$ per g dry egg mass $(\mathrm{n}=3)$ for the first day of the reproduction trial in 15d-U84 and 15d-U1054 respectively. A mean value of $26 \mathrm{nmol}$ $\mathrm{U}$ per $\mathrm{g}$ dry egg mass $(\mathrm{n}=3)$ was recorded on the final day of $15 \mathrm{~d}-\mathrm{U} 84$. The model predictions deviate from these observations. For the $15 \mathrm{~d}-\mathrm{U} 84$ condition, predicted $\left\langle M_{Q}\right\rangle_{d}$ in eggs on the first day of the $15 \mathrm{~d}$ trial is around $200-300 \mathrm{nmol} \mathrm{g}{ }^{-1}$ and ranges from 11 to $52 \mathrm{nmol} \mathrm{g}^{-1}$ on the last day (depending on the replicate). 
The module assumes uptake is proportional to environmental concentration. But when we adjust the DEB model with the one compartment toxico-kinetic module to whole body residues provided in Barillet et al. (2005) and Barillet et al. (2011), we see that at $2100 \mathrm{nM} \mathrm{U}$, uptake should be very important but it hardly is compared to predictions (fig. 8C). When looking at the scatter in the data then only some individuals actually have really high $\left\langle M_{Q}\right\rangle_{w}$ (maybe even one replicate out of 10), while values in other replicates are comparable across conditions. This can be seen by the factor five difference in final $\left\langle M_{Q}\right\rangle_{d}$ between female 8 and female 10 in $37 \mathrm{~d}-\mathrm{U} 420$ (fig. 7 ) bearing in mind that their final $W_{w}$ and $L_{f}$ are quasi-identical: $845.5 \mathrm{mg} / 41.4$ $\mathrm{cm}$ and $894.5 \mathrm{mg} / 42.0 \mathrm{~cm}$ for female 8 and 10 respectively. We further illustrate this point by representing final $\left\langle M_{Q}\right\rangle_{w}$ of the ten individuals in the U-420 condition in Barillet et al. (2011) (fig. 9A). $\left\langle M_{Q}\right\rangle_{w}$ seems inversely related to condition $\left(W_{w} / L_{f}^{3}\right)$. We are able to explain part of this observed variability in bioaccumulation by differences in feeding (fig. 9B). Differences in initial $L_{f}$ and initial contribution of $E_{R}$ to total $W_{w}$ further exacerbate the variability in the signal (not shown).

Predicted bioaccumulation is sensitive to the incorporation of metabolic handling of starvation into the core DEB model. The starvation rules (resorption of $E_{R}$ and degradation of $V$ ) are responsible for the (predicted) extreme concentrations of non fed individuals (triangle symbol fig. 9B).

If we wanted predictions more in the neighbourhood of observed final $\left\langle M_{Q}\right\rangle_{d}$ for the four females in 37d-U84, predicted values would be far too high in the 37d-U420. We chose values of $P_{V d}$ and $\dot{k}_{e}$ which were also in line with the U84 and U420 conditions in Barillet et al. (2005) and Barillet et al. (2011) (fig. 8A,B,D and E). And are left with the discrepancies seen for females in 37d-U84 (fig. 7, first row) and for the U-2100 condition in Barillet et al. (2005) (fig. 8C,F).

The factor two difference in final $\left\langle M_{Q}\right\rangle_{w}$ for both conditions exposed to $420 \mathrm{nmol} \mathrm{U} \mathrm{l}^{-1}$ was nicely explained by dilution by growth, i.e. last term in eq. (2) (fig. 8B,E). But we assumed that average length of fish in U420 conditions by Barillet et al. (2005) was smaller than for Barillet et al. (2011) (table C.4). In the 2011 study mean wet mass increase over $20 \mathrm{~d}$ is in the order of $30 \mathrm{mg}$, while in the 2005 study it is in the order of $100 \mathrm{mg}$.

\subsection{Embryo and early juvenile}

A value of $f=0.3$ was estimated for embryos and early juveniles; initial energy in the egg was set to 1.7J (table C.4). Model predictions are compared with data from Bourrachot et al. (2008) (fig. 10). Uranium bioaccumulation could not be captured using the same set of toxico-kinetic parameters as for adults (table C.4, Online Appendix C). Embryo U biomass/water partition coefficient was estimated to be almost a factor 4000 lower than for adults. Predicted $\left\langle M_{Q}\right\rangle_{d}$ is in line with that observed (fig. 10A). The best fit for $W_{d}$ and final $L_{f}$ of embryos and early juveniles exposed to 84 and $1054 \mathrm{nM} \mathrm{U}$ was obtained assuming mode of action $(\mathrm{G})$ with $\left[M_{Q 0}^{\mathrm{G}}\right]=0 \mathrm{nmol}$ $\mathrm{cm}^{-3}$ and $\left[M_{Q T}^{\mathrm{G}}\right]=0.211 \mathrm{nmol} \mathrm{cm}{ }^{-3}$ (table C.4, fig. 10B,C). Thus the NEC for effects on growth in the exposure media is $0 \mathrm{nmol} \mathrm{U} \mathrm{l}^{-1}$. Mode of action (M) and (A) could not capture the importance of effects on growth in the embryo period. As hardly any feeding occurred, mode of action (A) would hardly impact early juvenile growth. As structure is small during embryo and post embryo development, mode of action (M) entails small deviations of cumulative energy 


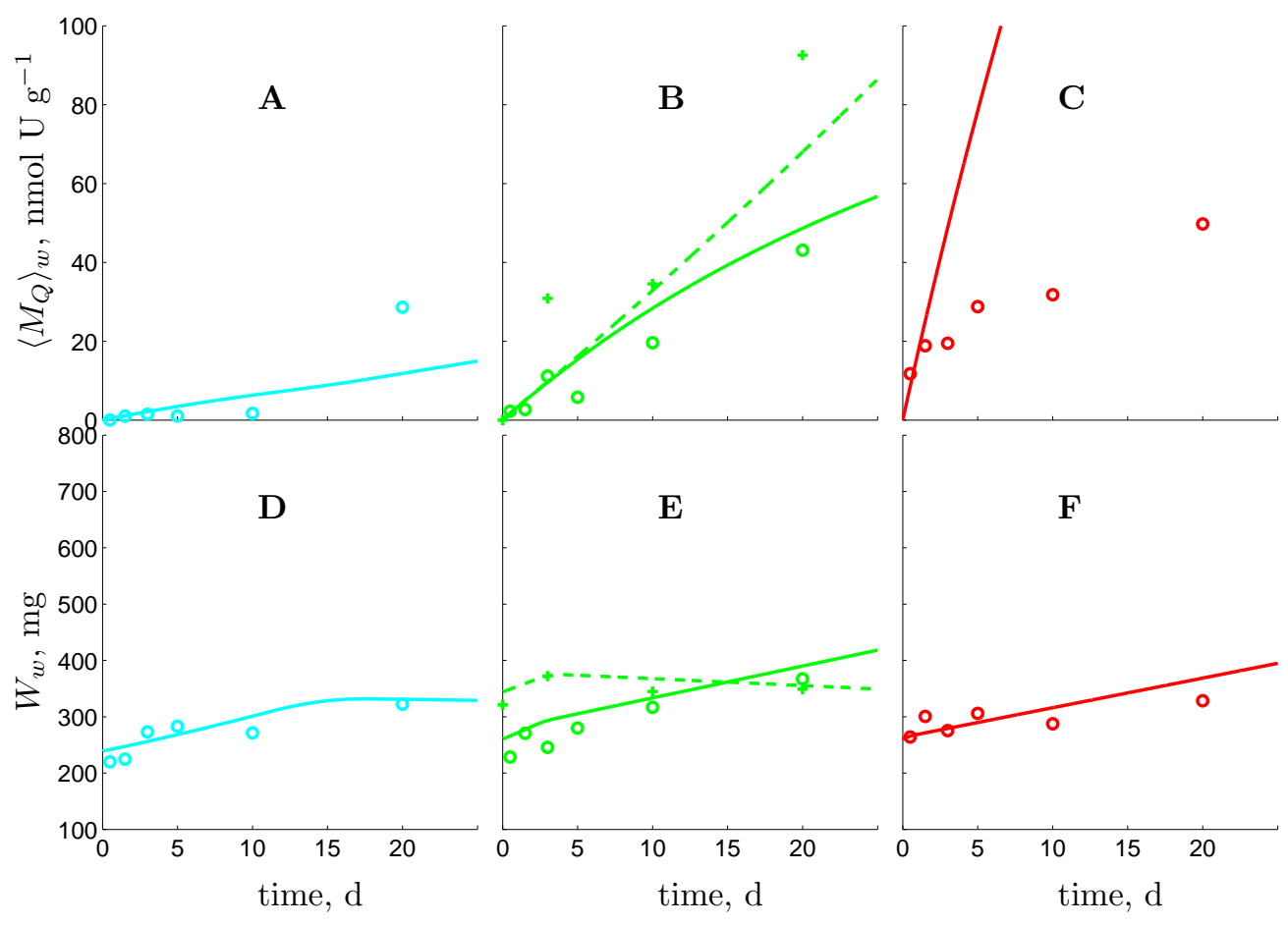

Figure 8: DEB model fits to adult bioaccumulation data. Circles: data published in Barillet et al. (2005); crosses: data published in Barillet et al. (2011). Solid lines: DEB model predictions for Barillet et al. (2005); dotted lines: DEB model predictions for Barillet et al. (2011). Parameters for each data set are in table C.4. (A), (B) and (C): mean internal concentration $\left\langle M_{Q}\right\rangle_{w}$, nmol $\mathrm{U} \mathrm{g}^{-1}$ fresh mass $\left(W_{w}\right)$ for exposure conditions 84,420 and $2100 \mathrm{nmol} / \mathrm{l}$ respectively. (D), (E) and (F): mean $W_{w}, \mathrm{mg}$ for exposure conditions 84 and $420 \mathrm{nmol} / \mathrm{l}$ and $2100 \mathrm{nmol} / \mathrm{l}$ respectively.

investment in growth. As starvation is included, an increase in somatic maintenance would increase shrinking from starvation but a more detailed data set (mass against time for several individuals) would be needed to detect this type of effect. Effects on survival are modelled assuming that survival is the sum of contributing hazards, Eq. (4): we assume a hazard due to accidents of $\dot{h}_{\text {acc }}=0.02 \mathrm{~d}^{-1}$ which explains background control mortality and a killing rate of $\dot{b}_{\dagger}=0.375 \mathrm{~cm}^{3} \mathrm{nmol}^{-1} \mathrm{~d}^{-1}$ which quantifies hazard induced by uranium on organism survival, Eq. (5). The NEC for survival is estimated to be $0 \mathrm{nmol} \mathrm{U} \mathrm{l}^{-1}$ with with $\left[M_{Q 0}^{\mathrm{S}}\right]=0 \mathrm{nmol} \mathrm{\textrm {cm } ^ { - 3 }}$ (table C.4). Predictions are in harmony with observed survival (fig. 10D).

These results answer our first question: effects of uranium on embryo and early juveniles differs from that of adults. Furthermore, the same set of toxic-kinetic parameters cannot describe the dynamics of $\left[M_{Q}\right]$ in both the early and late life stages.

\section{Discussion}

\subsection{Insights on reproductive physiology}

We show that cumulated energy investment in reproduction can be quantified separately from behavioural effects on reproductive output in a bioenergetic context. Toxicants can modify 

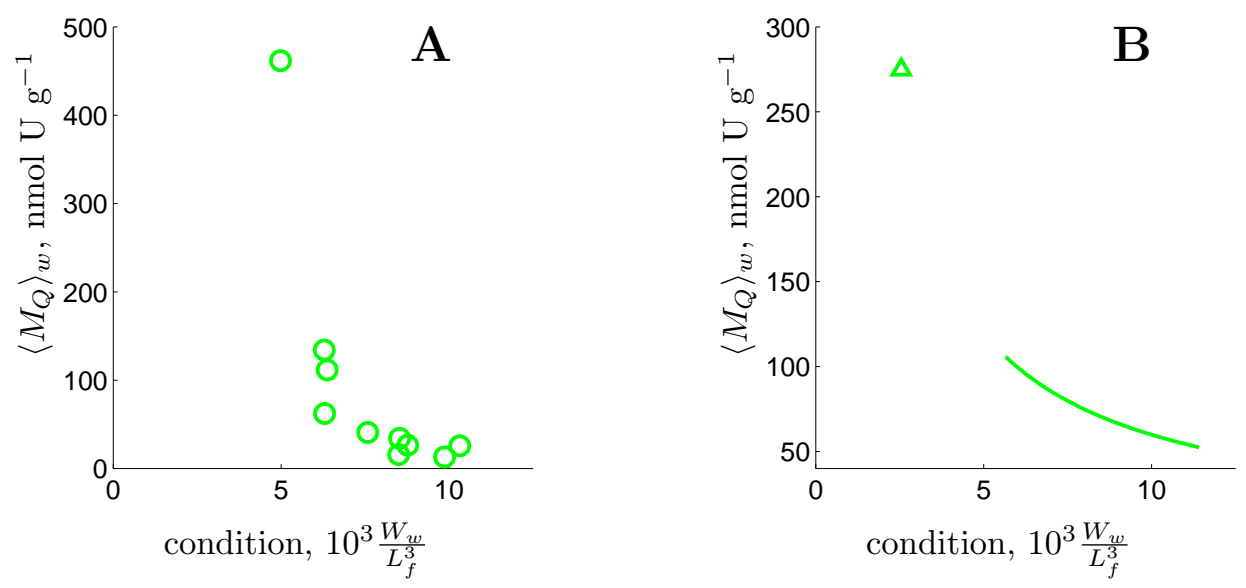

Figure 9: $\quad \mathrm{T}=25^{\circ} \mathrm{C}$. (A) Circles: data published in Barillet et al. (2011): internal concentration $\left\langle M_{Q}\right\rangle_{w}$, nmol $\mathrm{U} \mathrm{g}^{-1}$ fresh mass of the 10 individuals after $20 \mathrm{~d}$ of exposure to $420 \mathrm{nmol} \mathrm{U} / 1$. The extreme $\left\langle M_{Q}\right\rangle_{w}$ belongs to an individual with $L_{f}=2.9 \mathrm{~cm}$ and $W_{w}=121.5 \mathrm{mg}$. It is most likely a starving individual who has already started degrading structure to cover maintenance. (B) Solid line: DEB model predictions for internal concentration $\left\langle M_{Q}\right\rangle_{w}, \mathrm{nmol} \mathrm{U} \mathrm{g}^{-1} W_{w}$ of individuals after 20d of exposure to $420 \mathrm{nM}$ U. $L_{f}=3.6 \mathrm{~cm}$, negligible initial energy in reproduction buffer and no batch preparation. Computations are performed for values of $f$ ranging from 0.5 to 0.7. If we compute ultimate $\left\langle M_{Q}\right\rangle_{w}$ for a starved individual over the 20d period we obtain an extreme concentration (green triangle) right.

energetics (modification of a DEB model parameter), but can also modify behaviour. We offer the methodology to analyse both types of effects within a same DEB modelling framework.

This study further provides new scientific insight into reproductive physiology of zebrafish. Buffer handling rules turn out to be similar to multiple batch spawners such as Anchovy (Pecquerie et al., 2009). However, contrary to Anchovy batch preparation seems mainly food driven. We were surprised to see that in $15 \mathrm{~d}-\mathrm{U} 0$ batch preparation was either initiated at the onset of the $20 \mathrm{~d}$ accumulation, or during the $20 \mathrm{~d}$ accumulation and sometimes at the beginning of the reproduction trial (table C.3). Perhaps in the first case batch preparation was already triggered before the experiment. In the second case, maybe visual contact with males who were kept in neighbouring plexiglass aquaria sufficed. Further research is needed to understand the exact nature of the batch preparation trigger. Model fits to 15d-U0 (table C.3 and fig. E.6, Online Appendix E) show that there may indeed be an important behavioural component to triggering batch preparation. A physiological interpretation of $t_{b}$ (time at which batch preparation is triggered) could be the onset of ovulation.

In line with Pecquerie et al. (2009), inhibition of batch preparation seems to be triggered internally and we found the internal trigger to be when energy is taken from the buffer to cover somatic maintenance. This study shows that this is most likely the mechanism through which uranium inhibits spawning.

Effects of sensory organs and/ or behaviour should be quantifiable via effects on $\left[E_{B}\right]$ or $t_{b}$. We found that $\left[E_{B}\right]$ comports a lot of inherent scatter already in controls. In addition $\left[E_{B}\right]$ cannot be estimated when females do not spawn as is the case in 15d-U1054 and 37d-U420. Thus we are unable to conclude on absence or presence of an effect on $\left[E_{B}\right]$. 

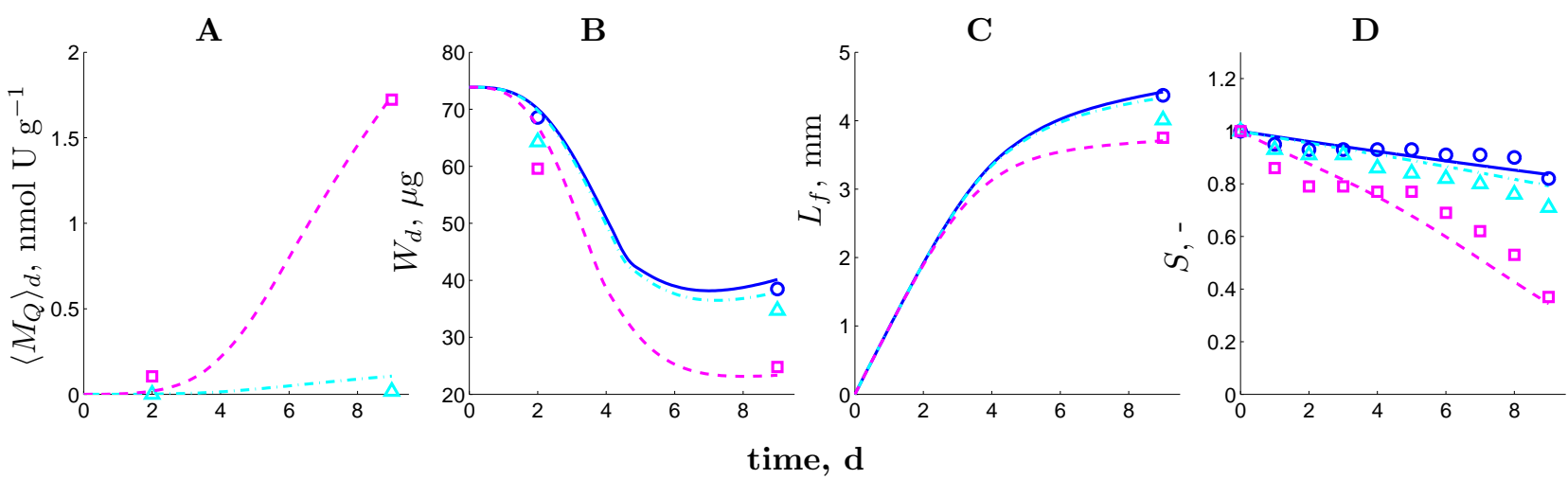

Figure 10: Symbols: data published in Bourrachot et al. (2008). $\mathrm{T}=25^{\circ} \mathrm{C}, f=0.3$. Lines DEB model predictions. Circles: control; triangles: exposed to $84 \mathrm{nM} \mathrm{U}$; squares: exposed to $1054 \mathrm{nM} \mathrm{U}$; solid lines: predictions for control; dot-dashed lines: predictions for $84 \mathrm{nM} \mathrm{U}$; dashed line: predictions for $1054 \mathrm{nM}$ U. A: Internal concentration: $\left\langle M_{Q}\right\rangle_{d}$, nmol $\mathrm{U} \mathrm{g}^{-1}$ dry mass, B: Dry mass $\left(W_{d}\right), \mu \mathrm{g}, \mathrm{C}$ : physical length $L_{f}$ and D: survival fraction $S$. Parameter values and initial conditions are in table C.4, Online Appendix C.

With more detailed mass measurements in combination with $N$, then contributions of $E_{R}$ to total biomass can be quite accurately quantified; assuming of course that a robust set of DEB parameters for the species is available. Performing mass and energy balancing on ripe and unripe buffer is a bit trickier, but after this first study, it also seems possible if well thought out experiments are performed. The 15d trial protocol (Bourrachot, 2009) is actually a good basis for designing such a study. Perhaps a clearer physiological link between ripe batch material and histological tissue analysis could be made in the future (e.g. linking batch preparation to oocyte maturation).

In this study, the total (live + dead) observed number of eggs $N$ was used to compare model predictions to observations. Observed dead eggs in this study comprise three types: (i) non fertilized but otherwise healthy, (ii) coagulated, and (iii) small totally opaque white spheres. We are not sure about the physiological nature of the latter two categories of dead eggs and understanding its dynamics was beyond the scope of this study. However we would like to highlight an interesting possibility for future research venues wishing to link sub-organismal to organismal responses: the proportion of dead eggs of type (ii) and (iii) in a batch may be related to residence time of ripe/ripening batch material inside the body.

Our results lend support to the role of maintenance as a demand system. The results empirically support an underlying assumption of DEB theory: that supply is surface area controlled and demand is volume linked (Kooijman, 2010). The theory would predict that fish close to ultimate size (as in the $37 \mathrm{~d}$ experiment) would need higher $f$ to cover somatic maintenance than individuals of smaller size. The sensitivity of fish to value of initial structure and $f$ in the $37 \mathrm{~d}$ experiment relative to the $15 \mathrm{~d}$ experiment with the smaller size class is indeed remarkable. Basically, fish in the $37 \mathrm{~d}$ experiment need at least $f=0.75$ to spawn and sometimes need $f \geq 0.8$, whereas in the $15 \mathrm{~d}$ experiment some fish can spawn with less than $f=0.7$. 


\subsection{Reproductive toxicology}

When looking at mean cumulated $N$ in 37d-U84 (fig. 3C) relative to control, a first interpretation would be a hormesis phenomenon. Accounting for physiological differences between females and ingestion levels $f$, then no hormesis is observed. Secondary stress induced through uranium might have increased ingestion for individuals in 37d-U84 and 37d-U420 who manifestly have much higher values of $f$ than controls. This was observed for Daphnia magna (Kooijman, 2010, Chap.6). In fact we even visually observed the much higher appetite in conditions 37d-U84 and 37d-U420 during the 37d exposure. Food availability is a major factor regulating effects (Kooijman, 1991; Jager et al., 2004; Zimmer et al., 2012)

On the basis of the $37 \mathrm{~d}-\mathrm{U} 84$ data, we explained effects on reproductive output as an increase in buffer material being burned to fuel maintenance processes. The increase in demand driven metabolic work must have an upper limit in the concentration ranges where survival is not impacted. We propose an upper limit to the increase in the neighbourhood of $1000 \mathrm{~J} \mathrm{~d}^{-1} \mathrm{~cm}^{-3}$, but further research is needed to test this.

Although we assumed the mode of action is an increase in the value of $\left[\dot{p}_{M}\right]$, more detailed physiological studies might better distinguish between an eventual effect on assimilation. An implied model property is that if $\mathrm{O}_{2}$ consumption were measured, then decreasing $\left\{\dot{p}_{A m}\right\}$ would decrease $\mathrm{O}_{2}$ consumption while increasing $\left[\dot{p}_{M}\right]$ would increase it.

If the targeted metabolic process is assimilation, it would not modify the conclusion that the mechanism behind inhibiting spawning is the process where energy is taken from the buffer

to cover somatic maintenance. Analysis of uranium toxicity data on Daphnia magna using DEB theory concluded that assimilation decreased in response to water-borne exposure to U (Massarin et al., 2010, 2011). Their study showed that effects of uranium on growth and reproduction were captured equally well when assuming a decrease in assimilation or an increase in somatic maintenance in the first two generations, but that effects on the third generation were better characterized by a decrease in assimilation. The authors used this last piece of evidence in combination with histological analysis of uranium induced damage to the gut wall to favour mode of action (A). A complementary study also reveals uranium induced damage to the gut wall in zebrafish (Augustine et al., 2012). More specifically there was a loss of gut wall architecture, presence of large necrotic zones and an overall decreases in gut bacteria.

It would be possible to obtain adequate fits for modes of actions (M) and (A) for the $15 \mathrm{~d}$ reproduction trials by Bourrachot (2009) as well as for all the females in 37d-U420. And it is indeed tempting to use histological analysis to tip the balance in favour of a decrease in assimilation. The deciding factor lies in the reproductive output of the females in the 37d-U84 condition. A decrease in assimilation cannot satisfactorily match the data. Somehow too much material in the reproduction buffer in being burned. An increase in $\left[\dot{p}_{M}\right]$ does however explain the phenomena. Uranium not only induces damage to the digestive tract, but also to the gills, liver, muscle, brain and gonads (Lerebours et al., 2010a,b; Barillet et al., 2010). Each organ comprises both reserve and structure. While it is tempting to at least delimit the reproduction buffer reserve material to the gonad, there is no satisfactory proof that even this is an accurate approximation. 


\subsection{Effects on growth}

This study also shows that uranium can target two separate metabolic processes in zebrafish, but further research is needed to understand how the two modes of action act together upon the organism. The contribution of initial amount of reproductive material to total biomass at the onset of adult exposure experiments in combination with negligible growth hampers firm conclusions about effects on growth when analysing adult data sets. However, uranium clearly impacts embryo and early juvenile growth (Bourrachot et al., 2008), fig. 10. Exposure to uranium contaminated lake effluent also negatively impacted growth of early juvenile green frogs Rana perezi (Marques et al., 2008). During the early life stages, growth may be a more sensitive endpoint than survival for evaluating uranium induced effects, although few toxicity studies address this problem. Since our results suggest a no effect concentration of 0 for effects on growth, this should be corrected for in future risk assessment studies.

Absence of observed effects on growth in adult fish (Cooley et al., 2000; Barillet et al., 2011) can be explained by the capacity of adults to resorbe material in the reproduction buffer to cover somatic maintenance. Independent empirical support for this assertion is that no significant mortality was observed when adult zebrafish were starved for $70 \mathrm{~d}$ (pers. comm. X. Cousin). The analysis of adult data sets alone, would have been quite misleading since we would have concluded that there are no effects on growth. The model predicts that combining increasing $\left[E_{G}\right]$ and $\left[\dot{p}_{M}\right]$ would lead to severe effects on both growth and reproduction if young juveniles were exposed all the way to puberty. Survival of juveniles should also be more impacted relative to adults because the latter have the possibility to draw on reproductive material to cover somatic maintenance; juveniles do not.

\subsection{Elimination and uptake}

This study also shows that elimination through reproduction explains why reproductive output was severely impacted in the study by Bourrachot (2009) but not in the 37d trial. Empirical support for the detoxifying role of reproduction is in the sensitivity of effects relative to value of batch energy density. Female 10 was not impacted but females 2 and 3 were in $37 d-U 84$. We were surprised to see that effects on metabolism were so sensitive to batch energy density and wonder again at what factors play an important role in the value of $\left[E_{B}\right]$.

By accounting for elimination through reproduction, qualitative trends in accumulation and depuration are captured (Bourrachot, 2009). Nonetheless, the overall capacity of zebrafish to (totally) eliminate uranium still remains an open question. Uranium was not entirely eliminated in muscle, liver, gills or brain after a week (Lerebours et al., 2009) nor in whole body residues after 30 days (Labrot et al., 1999). However, uranium was eliminated from muscle tissue of goldfish Carassius auratus previously exposed for 4 days (Lourenço et al., 2010). After 6 months of depuration, humans previously exposed to mean values of $2.6 \mathrm{mM} \mathrm{U}$ in their drinking water still had high levels of $U$ in their urine (Orloff et al., 2004). However, U was efficiently eliminated in the annelid Eisenia fetida (Labrot et al., 1999) and the arthropod Hyalella azteca (Alves et al., 2009).

We made the simplifying assumption that the only uptake route is through the water. Uptake can also occur through food and also be transferred maternally to offspring (Simon et al., 2011). In experimental settings, as analysed here, food (Tetramin ${ }^{\mathrm{TM}}$ ) is not spiked but 
may contain background levels of $\mathrm{U}\left(\approx 2.6 \mathrm{nmol} \mathrm{U} \mathrm{g}^{-1}\right.$ Tetramin $^{\mathrm{TM}}, \mathrm{K}$. Faucher pers. comm. $)$ as well as may contain uranium adsorbed from the water column between reaching the water and being eaten. Care was always taken to reduce that time interval, but control $\left\langle M_{Q}\right\rangle_{d}$ presents background levels of uranium (not shown).

\subsection{On the origin of variability in bioaccumulation measurements}

Using DEB theory, internalized PCB was correlated to prior feeding history for many individuals in a study involving the common sole, Solea solea (Eichinger et al., 2012). Growth trajectories of each individual were found to differ considerably; differences in feeding history were quantified by estimating individual values of $f$. Very importantly, the authors demonstrate that using the mean $f$ value from all the sampled individuals does not always provide accurate predictions for data expressed as the average observed values. Our study is fully in line with that result. We further contribute to this insight by showing that not only does feeding history contribute to the variability, but so does initial amount of reproduction buffer material. In some instances, such as the $15 \mathrm{~d}$ reproduction trial, even the initial amount of ripe batch material relative to total available reproduction buffer material matters for interpreting results.

A high percentage of variability in accumulation can be explained by energetics, but not all. Uranium showed the same propensity to accumulate in an extreme manner in certain but not all replicates of Corbicula fluminea. The inter-individual variability in bioaccumulation was very much reduced for Corbicula fluminea exposed to selenium (Adam, 2006, pp.40). A prior study on uranium bioaccumulation in zebrafish brain and muscle tissue also recorded the same high inter-individual variability (Lerebours et al., 2010a). This pattern holds for actinide uptake in general for the mussel Mytilus edulis (Lobel et al., 1991). Future studies might remove variability in initial conditions of fish in each exposure condition and compare bioaccumulation across groups of adults of different classes of initial conditions to a same exposure condition to more accurately determine dynamics of uptake and elimination.

\subsection{Deviations from the extended one compartment toxico-kinetic model}

In this study effects were taken proportional to total density of $U$ in the body expressed as nmol $\mathrm{U} \mathrm{cm}^{-3}$ structure. Since only the aqueous fraction of internalized $\mathrm{U}$ may be actively incurring damage to molecules (i.e. DNA), it may be relevant to take effects proportional to density of $\mathrm{U}$ in structure only: $M_{Q V} / V$ (Muller and Nisbet, 1997). This would help explain how growth of F1 embryos and early juveniles, who inherited $U$ from the mother and were raised in clean water, was hardly impacted (Bourrachot, 2009). On the other hand it may also be analytically impossible to measure the aqueous fraction in whole body residues.

A very enigmatic aspect is the systematic presence of individuals with extreme $\left\langle M_{Q}\right\rangle_{d}$ relative to other replicates (fig. 9A and 7). What could be the energetic difference between female 8 and 10 in the U-420 condition that could promote a factor 5 difference in accumulation (fig. 7)? We compared acclimation history of both females and found that in the $35 \mathrm{~d}$ acclimation to individual feeding and reproduction, female 8 never reproduced while female 10 reproduced once: 574 eggs were spawned the day before exposure. We further compared lateral and dorsal 
portraits of both individuals at exposure day 3 (fig. D.4, Appendix D). Both fish look quasiidentical, however the 574 eggs spawned by female 10 represents a loss of approximately 280 $\mathrm{mg}, W_{w}$ (assuming $E_{0}=1.7 \mathrm{~J}$ ). If we assume $W_{w}$ is similar at time of portrait then the difference between female 8 and 10 is in reserve density. In this case $[E]$ for female 8 must have been less important than for female 10 at the onset of exposure. This is suggestive of a link between between reserve density and bioaccumulation. A possible hypothesis is that $\mathrm{U}$ binds (maybe even irreversibly) with receptors in $E$. Such receptors could represent a constant fraction of $E$. When comparing between individuals, higher $[E]$ would entail more fixation sites. A process potentially involving precipitating uranium in specialized vacuoles (Pereira et al., 2012) might also be involved in detoxification bringing up internal concentrations but lowering the internal fraction of active compound which induces effects.

Embryo and adult toxico-kinetic parameters seem to differ. This may be due to the very sparse data sets but may also be linked to different surface area volume relationships in the early juvenile and the adult. Take for instance ratio of gill surface to total surface: adults have more gill surface per total surface that juveniles. We aimed for maximum simplicity: leading to the simplest formulation possible of toxico-kinetics (Kooijman, 2010). The chorion is assumed to be part of the overheads of growth (quantified by $\kappa_{R}$ ) and Bourrachot (2009) has demonstrated that a small part of uranium ends up in the chorion in the case of maternal transfer to offspring. We also did not account for adsorption to the chorion during direct exposure to offspring which was also demonstrated to occur (Bourrachot et al., 2008). It is capital to link any toxico-kinetic module with the energetics of the organism (e.g. van Haren et al., 1994; Bodiguel et al., 2009; $\mathrm{Ng}$ and Gray, 2009; Eichinger et al., 2010). However the details around how water chemistry (e.g. $\mathrm{pH}$ ) affects ionisation which in turn modulates the amount of uranyl ion (thought to be the most bioavailable (Fortin et al., 2007)) has not been addressed in this study. Natural extensions can be built to deal with water chemistry for operational purposes (Kooijman, 2010, Chap.6).

\subsection{Perspectives}

Future studies might consider presenting data as individual mass and reproductive output. Monte Carlo simulation methods where uncertainty is placed on behaviourally controlled parameters $\left(f, t_{b},\left[E_{B}\right]\right)$ might be developed to estimate toxicity parameters for each individual separately. Perhaps taking differences in initials conditions and buffer handling rules in combination with perfecting experimental protocols to reduce inter-individual variability in ingestion might allow for even more accurate quantification of very subtle sub-lethal effects on metabolism. In future experiments smaller adults should be preferred to individuals who are close to ultimate size to reduce inter spawn interval and sensitivity of spawning to small behavioural differences in ingestion. The biggest weakness in all the data sets is absence of initial measurements of mass and length. This happily can very easily be corrected in future experiment protocols. It would be possible to extend the same modelling framework to understand effects of ionizing radiation relative to depleted uranium. For instance, Bourrachot et al. (2008) show that ionizing radiation are seemingly on development in combination with survival, but not on growth which is in stark contrast to effects of depleted uranium alone. Effects on maturation (quantified by the life stage parameters) would emulate this type of effect. 


\section{Concluding remarks}

We show here that it is possible to analyse toxicity data from a number of separate experiments using DEB model parameters previously estimated using independent eco-physiological data on the species. Zebrafish is an OECD test species and so there is now a unifying framework for analysing effects of any number of chemicals considering a same DEB parameter set for the control. This will allow impartial evaluation of effects of feeding on results and reinforce robustness for each of the studies. This study further provides a handle for analysing behavioural data (spawning and/or feeding) using an individual based bio-energetic model. Behavioural response to toxicants are a very ecologically relevant response.

Finally, the random sampling strategy seems really counter-productive in a laboratory setting. A lot of the differences between individuals seems to be in size, mass, relative contributions of reserve and reproductive material to total wet mass and maybe even how much of the reproductive material is ripe batch material at the time of sampling. These types of differences are not important and are simply random chance events (artefacts). Comparing mean values of measurable quantities at each sampling time amounts to assuming that the distribution of each one of the parameters which quantifies each one of these differences is identical and has same parameters (e.g. same mean and standard deviation assuming normal distribution) at each time point. What we hoped to have illustrated through this study is that while size, mass, relative contributions of reserve and reproductive material to total wet mass, spawning behaviour etc. may be (very) different in each of the replicates in a condition, the cumulated reproductive investment and cumulative mass investment in growth during the experiment is quantifiable for each individual. Whatever scientific philosophy is adopted, we will all agree that it is the quantification of these last two physiological processes which are of primary concern in ecotoxicology.

\section{Acknowledgements}

This work is part of the ENVIRHOM research program supported by the Institute for Radioprotection and Nuclear Safety and the Provence Alpes Côte d'Azur region. We are grateful to Stephanie Bourrachot for providing raw data from her thesis, to Virginie Camilleri for ICP-AES analysis and technical insight into how to maintain a constant exposure level of U over 37 days, to Sylvie Pierrisnard for ionic chromatography analysis, to Alban Carlat for assisting in data collection and to François Brion and Alexandre Péry for helpful comments during experimental design phase. The comments of two anonymous reviewers and the editor greatly improved this Manuscript.

\section{References}

Adam, C. 2006. Indicateurs de l'exposition et des effets des radionucléides dans les ecosystèmes aquatiques continentaux. HDR, Université Aix-Marseille I.

Alves, L., Borgmann, U., and Dixon, D. 2009. Kinetics of uranium uptake in soft water and the effect of body size, bioaccumulation and toxicity to Hyalella azteca. Env Poll, 157(8-9):2239-2247. 
Augustine, S., Gagnaire, B., Adam-Guillermin, and Kooijman, S. A. L. M. 2011a. Developmental energetics of zebrafish, Danio rerio. Comp Biochem Physiol A, 159:275-283.

Augustine, S., Litvak, M. K., and Kooijman, S. A. L. M. 2011b. Stochastic feeding of teleost fish and their metabolic handling of starvation. J Sea Res, 66:411-418.

Augustine, S., Pereira, S., Gagnaire, B., Floriani, M., Camilleri, V., Adam-Guillermin, C., and Kooijman, S. A. L. M. 2012. Uranium induced alteration of gut wall histology in zebrafish, Danio rerio. in prep.

Barillet, S., Adam, C., Palluel, O., M., P. J., and Devaux, A. 2011. Uranium bioaccumulation and biological disorders induced in zebrafish (Danio rerio) after a depleted uranium waterborne exposure. Env Poll, 159:495502 .

Barillet, S., Buet, A., Adam, C., and Devaux, A. 2005. Does uranium exposure induce genotoxicity in the teleostean Danio rerio? First experimental results. Radioprotection, 40:S175-S181.

Barillet, S., Larno, V., Floriani, M., Devaux, A., and Adam, C. 2010. Ultrastructural effects on gill, muscle, and gonadal tissues induced in zebrafish (Danio rerio) by a waterborne uranium exposure. Aquat Toxicol, 100:295-302.

Bodiguel, X., Maury, O., Mellon-Duval, C., Roupsard, F., Le Guellec, A. M., and Loizeau, V. 2009. A dynamic and mechanistic model of PCB bioaccumulation in the European hake (Merluccius merluccius). J Sea Res, $62: 124-134$.

Bourrachot, S. 2009. Etude des effets biologique de l'exposition à l'uranium chez le poisson zèbre (D. rerio). Impact sur les stades de vie. PhD thesis, L’Université Aix-Marseille I - Université de Provence.

Bourrachot, S., Simon, O., and Gilbin, R. 2008. The effects of waterborne uranium on the hatching success, development, and survival of early life stages of zebrafish (Danio rerio). Aquat Toxicol, 90:29-36.

Cooley, H. M., Evans, R. E., and Klaverkamp, J. F. 2000. Toxicology of dietary uranium in lake whitefish (Coregonus clupeaformis). Aquat Toxicol, 48:495-515.

Eichinger, M., Loizeau, V., Le Guellec, A. M., Gastineau, O., Roupsard, F., and Bacher, C. 2012. An individual modelling approach to estimate polychlorinated biphenyl (PCB) bioaccumulation and their potential effects on common sole growth. in prep.

Eichinger, M., Loizeau, V., Roupsard, F., Le Guellec, A., and Bacher, C. 2010. Modelling growth and bioaccumulation of Polychlorinated biphenyls in common sole (Solea solea). J Sea Res, 64(3):373 - 385.

Fernandes, H. M., Veiga, L. H. S., Franklin, M. R., Prado, V. C. S., and Taddei, J. F. 1995. Environmental impact assessment of uranium mining and milling facilities: A study case at the poços de caldas uranium mining and milling site, brazil. J Geochem Explor, 52:161-173.

Fortin, C., Denison, F. D., and Garnier-Laplace, J. 2007. Metal-Phytoplankton interactions: modeling the effect of competing ions $\left(\mathrm{H}^{+}, \mathrm{Ca}^{2+}\right.$, and $\left.\mathrm{Mg}^{2+}\right)$ on uranium uptake. Envir Toxicol Chem, 26(2):242-248.

Fortin, C., Dutel, L., and Garnier-Laplace, J. 2004. Uranium complexation and uptake by a green alga in relation to chemical speciation: the importance of the free uranyl ion. Envir Toxicol Chem, 23:974-981.

Gerlach, G. 2006. Pheromonal regulation of reproductive success in female zebrafish: female suppression and male enhancement. Anim Behav, 72:1119-1124.

Hill, A. J., Teraoka, H., Heideman, W., and Peterson, R. E. 2005. Zebrafish as a model vertebrate for investigating chemical toxicity. Toxicol Sci, 86(1):6-19. 
Jager, T., Crommentuijn, T., Van Gestel, C. A. M., and Kooijman, S. A. L. M. 2004. Simultaneous modeling of multiple end points in life-cycle toxicity tests. Environ Sci Technol, 38:2894-2900.

Jager, T., Heugens, E. H. W., and Kooijman, S. A. L. M. 2006. Making sense of ecotoxicological test results: towards application of process-based models. Ecotoxicology, 15:305-314.

Jager, T. and Klok, C. 2010. Extrapolating toxic effects on individuals to the population level: the role of dynamic energy budgets. Philos Trans R Soc B, 365:3531-3540.

Jager, T., Vandenbrouck, T., Baas, J., De Coen, W. M., and Kooijman, S. A. L. M. 2010. A biology based approach for mixture toxicity of multiple endpoints over the life cycle. Ecotoxicology, 19:351-361.

Jager, T. and Zimmer, E. I. 2012. Simplified dynamic energy budget model for analysing ecotoxicity data. Ecol Model, 225:74-81.

Jurgens, B. C., Fram, M. S., Belitz, K., Burow, K. R., and Landon, M. K. 2010. Effects of Groundwater Development on Uranium: Central Valley, California, USA. Ground Water, 48(6):913-928.

Kooijman, S. A. L. M. 1981. Parametric analysis of mortality rates in bioassays. Wat Res, 15:107-119.

Kooijman, S. A. L. M. 1991. Effects of feeding conditions on toxicity for the purpose of extrapolation. Comp Biochem Physiol C, 100C(1/2):305-310.

Kooijman, S. A. L. M. 2009. What the egg can tell us about its hen: Embryonic development on the basis of dynamic energy budgets. J Math Biol, 58(3):377-394.

Kooijman, S. A. L. M. 2010. Dynamic Energy Budget Theory for metabolic organization. Cambridge.

Kooijman, S. A. L. M. and Bedaux, J. J. M. 1996. The analysis of aquatic toxicity data. VU University Press, Amsterdam. ISBN 90-5383-477-X.

Kooijman, S. A. L. M., Pecquerie, L., Augustine, S., and Jusup, M. 2011. Scenarios for acceleration in fish development and the role of metamorphosis. J Sea Res, 66:419-423.

Kooijman, S. A. L. M., Sousa, T., Pecquerie, L., Van der Meer, J., and Jager, T. 2008. From food-dependent statistics to metabolic parameters, a practical guide to the use of dynamic energy budget theory. Biol Rev, $83(4): 533-552$.

Kooijman, S. A. L. M. and van Haren, R. J. F. 1990. Animal energy budgets affect the kinetics of xenobiotics. Chemosphere, 21(4-5):681-693.

Laale, H. W. 1977. The biology and use of zebrafish, Brachydanio rerio in fisheries research. J Fish Biol, $10: 121-173$

Labrot, F., Narbonne, J. F., Ville, P., Saint Denis, M., and Ribera, D. 1999. Acute Toxicity, Toxicokinetics, and Tissue Target of Lead and Uranium in the Clam Corbicula fluminea and the Worm Eisenia fetida: Comparison with the Fish Brachydanio rerio. Arch Environ Con Tox, 36:167-178.

Lawrence, C. 2007. The husbandry of zebrafish (Danio rerio) : a review. Aquaculture, 269:1-20.

Lerebours, A., Adam-Guillermin, C., Brèthes, D., Frelon, S., Floriani, M., Camilleri, V., Garnier-Laplace, J., and Bourdineaud, J.-P. 2010a. Mitochondrial energetic metabolism perturbations in skeletal muscles and brain of zebrafish (Danio rerio) exposed to low concentrations of waterborne uranium. Aquat Toxicol, 100:66-74. 
Lerebours, A., Bourdineaud, J.-P., Van Der Ven, K., Vandenbrouck, T., Gonzalez, P., Camilleri, V., Floriani, M., Garnier-Laplace, J., and Adam-Guillermin, C. 2010b. Sublethal effects of waterborne uranium exposures on the zebrafish brain: Transcriptional responses and alterations of the olfactory bulb ultrastructure. Environ Sci Technol, 44(4):1438-1443.

Lerebours, A., Gonzalez, P., Adam-Guillermine, C., Camilleri, V., Bourdineaud, J.-P., and Garnier-Laplace, J. 2009. Comparative analysis of gene expression in brain, liver, skeletal muscles, and gills of zebrafish (Danio rerio) exposed to environmentally relevant waterborne uranium concentrations. Envir Toxicol Chem, 28(6):1271-1278.

Lika, K., Freitas, V., van der Veer, H. W., van der Meer, J., Wijsman, J. W. M., Pecquerie, L., Kearney, M. R., and Kooijman, S. A. L. M. 2011a. The "covariation method" for estimating the parameters of the standard Dynamic Energy Budget model I: philosophy and approach. J Sea Res, 66:270-277.

Lika, K., Kearney, M. R., and Kooijman, S. A. L. M. 2011b. The "covariation method" for estimating the parameters of the standard Dynamic Energy Budget model II: properties and preliminary patterns. J Sea Res, 66:278-288.

Lobel, P. P., Longuerich, H., Jackson, S. E., and Belkhode, S. 1991. A major factor contributing to the high degree of unexplained variability of some elements concentrations in biological tissue: 27 elements in 5 organs of the mussel Mytilus as a model. Arch Environ Con Tox, 21:118-125.

Lourenço, J., Castro, B., Machado, R., Nunes, B., Mendo, S., Gonçcalves, F., and Pereira, R. 2010. Genetic, biochemical, and individual responses of the teleost fish Carassius auratus to uranium. Arch Environ Con Tox, 58(4):1023-1031.

Marques, S., Gonçalves, F., and Pereira, R. 2008. Effects of a uranium mine effluent in the early-life stages of Rana perezi Seoane. Sci Tot Environ, 402(1):29-35.

Massarin, S., Alonzo, F., Garcia-Sanchez, L., Gilbin, R. Garnier-Laplace, J., and Poggiale, J.-C. 2010. Effects of chronic uranium exposure on life history and physiology of Daphnia magna over three successive generations. Aquat Toxicol, 99(3):309-319.

Massarin, S., Beaudouin, R., Zeman, F., Floriani, M., Gilbin, R., Alonzo, F., and Pery, A. R. R. 2011. Biologybased modeling to analyze uranium toxicity data on Daphnia magna in a multigeneration study. Environ Sci Technol, 45(9):4151-8.

Muller, E. and Nisbet, R. 1997. Modeling the effect of toxicants on the parameters of dynamic energy budget models. In Dwyer, F. J., Doane, T. R., and Hinman, M., editors, Environmental toxicology and risk assessment: modeling the risk assessment (sixth volume), ASTM STP 131\%. American society for testing and materials.

Ng, C. A. and Gray, K. A. 2009. Tracking bioaccumulation in aquatic organisms: A dynamic model integrating life history characteristics and environmental change. Ecol Model, 220(9-10):1266-1273.

Nuclear Energy Agency 2008. Uranium 200\%: Resources, Production and Demand. OECD Publishing.

Orloff, K. G., Mistry, K., Charp, P., Metcalf, S., Marino, R., Shelly, T., Melaro, E., Donohoe, A. M., and Jones, R. L. 2004. Human exposure to uranium in groundwater. Env Res, 94:319-326.

Pecquerie, L., Petitgas, P., and Kooijman, S. A. L. M. 2009. Modeling fish growth and reproduction in the context of the dynamic energy budget theory to predict environmental impact on anchovy spawning duration. J Sea Res, 62:93-105.

Pereira, S., Camilleri, V., Floriani, M., Cavalie, I., Garnier-Laplace, J., and Adam-Guillermin, C. 2012. Genotoxicity of uranium contamination in embryonic zebrafish cells. Aquat Toxicol, 109:11-16. 
Simon, O., Mottin, E., Geffroy, B., and Hinton, T. 2011. Effects of dietary uranium on reproductive endpoints - fecundity, survival, reproductive success - of the fish Danio rerio. Envir Toxicol Chem, 30(1):220-225.

Sousa, T., Domingos, T., Poggiale, J. C., and Koijman, S. A. L. 2010. Dynamic energy budget restores coherence in biology. Philos Trans $R$ Soc B, 365:3413-3428.

Spence, R., Gerlach, G., Lawrence, C., and Smith, C. 2008. The behaviour and ecology of the zebrafish, Danio rerio. Biol Rev, 83(1):13-34.

Uralbekov, B. M., Smodis, B., and Burkitbayev, M. 2011. Uranium in natural waters sampled within former uranium mining sites in Kazakhstan and Kyrgyzstan. J Radioanal Nucl Chem, 289:805-810.

van Haren, R. J. F., Schepers, H. E., and Kooijman, S. A. L. M. 1994. Dynamic energy budgets affect kinetics of xenobiotics of the marine mussel Mytilus edulis. Chemosphere, 29(2):163-189.

Villa, M., Manjón, G., Hurtado, S., and García-Tenorio, R. 2011. Uranium pollution in an estuary affected by pyrite acid mine drainage and releases of naturally occurring radioactive materials. Mar Poll Bull, 62:15211529.

Zimmer, E. I., Jager, T., Ducrot, V., and Kooijman, S. A. L. M. 2012. Juvenile food limitation in standard tests- ecotoxicologists, be warned! Ecotoxicology. To appear.

Information about the DEB research program and its results can be found at http://www . bio.vu.nl/thb/deb/. The Add_My_Pet collection can be found at http://www.bio.vu.nl/thb/deb/deblab/add_my_pet/. 


\section{A Physical and chemical composition of exposure media for $U$ toxicity experiments}

The experimental system used in our $37 \mathrm{~d}$ exposure experiment is fully described in Augustine et al. (2011a). Briefly, each of the five aquaria in a holding tank is equipped with a water distributor. Each distributor occupies a central position above an aquaria. We incorporated an internal recirculating pump (Hydor) inside the holding tank. Eheim hose connectors are used to link the pump to plastic hosing ensuring water arrival to each water distributor. The pump is equipped with a foam filter and continuously runs holding tank water through each individual water distributor ensuring homogeneous mixing of the holding tank water throughout each of the permeable aquaria between daily water renewal events. About $25 \%$ of holding tank water is removed daily (care is taken to remove all feces and debris at this stage) and fresh water is brought to the system. Air stones linked to air pumps and immersed thermostat resistances were used to maintain temperature and oxygen levels. Fresh water is kept in 100 or 300L reservoirs and drawn to the holding tank with an immersible pump (Hydor).

During acclimation the $\mathrm{pH}$ remained stable. During exposure $\mathrm{pH}$ was monitored continuously by $\mathrm{pH}$ regulators (Consort R301, Illkirch, Belgium) linked to a $\mathrm{pH}$ electrode and a peristaltic pump. This was carried out to avoid variations in $\mathrm{pH}$ in response to addition of $\mathrm{U}$ to water. The regulator switched on the peristaltic pump in the event the electrode recorded a $\mathrm{pH}$ below 6.4. The peristaltic pump transported $\mathrm{NaOH} 10^{-1} \mathrm{M}$ to exposure media several $\mathrm{cm}$ away from the $\mathrm{pH}$ electrode and away from aquaria. Electrodes were verified once daily by immersion into 2 buffer solutions $(\mathrm{pH}=4, \mathrm{pH}=7, \mathrm{VWR})$. $\mathrm{pH}$ was further measured on separate electrodes (VWR) which were calibrated every 3 days to test for consistency of measurements between both types of electrodes. After the first few days of exposure the regulator was rarely used and $\mathrm{pH}$ was stable even considering the continuous addition of $\mathrm{U}$ to the system via the capillaries.

During each feeding event granules were dispensed in small amounts to each individual until satiation. Furthermore, granules were dispensed slowly to limit contact of food with (contaminated) water. Water renewal always took place after morning feeding. Weekly supplements of spirulina and/or live Daphnia magna were given.

Measured concentrations of $\mathrm{U}$ in the exposure media during the $37 \mathrm{~d}$ of exposure are presented in fig. A.1. Further details on the composition of the synthetic water and how the experimental protocol relates to the literature-based data we analyse can be found in table A.1. 


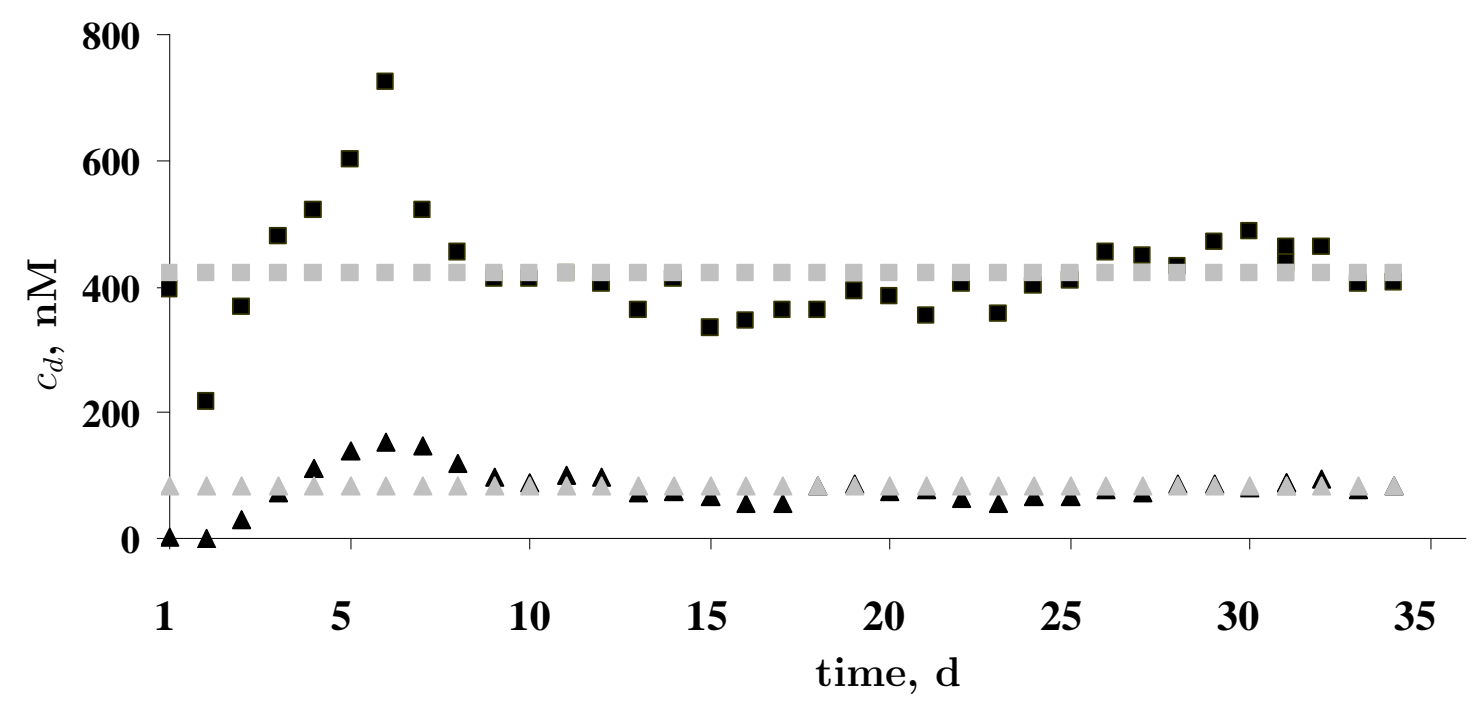

Figure A.1: Concentration of $\mathrm{U}$ in exposure media during $37 \mathrm{~d}$ accumulation and reproduction experiment. Black squares: ACP-AES measurements of U concentration, $c_{d} \mathrm{nM}$, in $420 \mathrm{nM}$ condition; grey squares: nominal concentration of $420 \mathrm{nM}$ condition. Black triangles: ICP-AES measurements of $c_{d}$ in $84 \mathrm{nM}$ condition; grey triangles: nominal concentration in $84 \mathrm{nM}$ condition. 
Table A.1: Compilation of physical and chemical parameters of all the studies analysed using the DEB model. We only consider the first 9 days of exposure in the embryo and early juvenile experiment by Bourrachot (2009), but the authors continue the experiment until 15d (see text for explanation). $\mathrm{pH}$, temperature $(\mathrm{T})$ and uranium $(\mathrm{U})$ correspond to nominal experiment values; see text for mean value \pm standard deviation for $\mathrm{pH}$ and $\mathrm{T}$ and fig. A.1 for measured $\mathrm{U}$ concentration (ICP-AES) for this study.

\begin{tabular}{|c|c|c|c|c|c|}
\hline & this study & $\begin{array}{c}\text { (Barillet et al., } \\
2005)\end{array}$ & $\begin{array}{c}\text { (Barillet et al., } \\
\text { 2011) }\end{array}$ & $\begin{array}{l}\text { (Bourrachot } \\
\text { et al., 2008) }\end{array}$ & $\begin{array}{c}\text { (Bourrachot, } \\
\text { 2009) }\end{array}$ \\
\hline life stage & adult & adult & adult & $\begin{array}{c}\text { embryo + early } \\
\text { juvenile }\end{array}$ & adult \\
\hline $\begin{array}{c}\text { exposure } \\
\text { duration }(\mathrm{d})\end{array}$ & 37 & 20 & 20 & 9 & 20 \\
\hline $\begin{array}{l}\text { depuration } \\
\text { duration }(\mathrm{d})\end{array}$ & no & no & no & no & 15 \\
\hline feeding & $\begin{array}{c}\text { Tetramin }^{\mathrm{TM}} \\
\text { granules } A d \\
\text { libitum } 2-3 \mathrm{x} \mathrm{d}\end{array}$ & $\begin{array}{c}\text { standard fish } \\
\text { pellets, } 1 \% \\
\text { body mass per } \\
\text { day }\end{array}$ & $\begin{array}{c}\text { standard fish } \\
\text { pellets, } 1 \% \\
\text { body mass per } \\
\text { day }\end{array}$ & no feeding & $\begin{array}{l}\text { tatramin }^{\mathrm{TM}} \\
\text { flakes } 2 \mathrm{x} d\end{array}$ \\
\hline individually fed & yes & no & no & no & no \\
\hline $\mathrm{K}^{+}(\mathrm{mM})$ & 0.15 & 0.16 & 0.16 & 0.15 & 0.15 \\
\hline $\mathrm{Na}^{+}(\mathrm{mM})$ & 0.32 & 0.50 & 0.50 & 0.32 & 0.32 \\
\hline $\left.\mathrm{Mg}^{2+}(\mathrm{mM})\right)$ & 0.20 & 0.20 & 0.20 & 0.19 & 0.19 \\
\hline $\mathrm{Ca}^{2+}(\mathrm{mM})$ & 0.29 & 0.29 & 0.29 & 0.29 & 0.29 \\
\hline $\mathrm{Cl}^{-}(\mathrm{mM})$ & 0.92 & 0.91 & 0.91 & 0.92 & 0.92 \\
\hline $\mathrm{NO}_{3}^{-}(\mathrm{mM})$ & 0.31 & 0.50 & 0.50 & 0.32 & 0.32 \\
\hline $\mathrm{SO}_{4}^{2-}(\mathrm{mM})$ & 0.10 & 0.10 & 0.10 & 0.10 & 0.10 \\
\hline $\mathrm{pH}$ & 6.5 & 6.5 & 6.5 & 6.5 & 6.5 \\
\hline $\mathrm{T}\left({ }^{\circ} \mathrm{C}\right)$ & 26 & 25 & 24 & 25 & 25 \\
\hline $\mathrm{U}(\mathrm{nM})$ & $0,84,420$ & $0,84,420,2100$ & 0,420 & $0,84,1054$ & $0,84,1054$ \\
\hline
\end{tabular}




\section{B DEB equations for modelling $U$ toxicity experiments}

The core DEB model equations are specified in table B.2. Additional terms are specified below. $\dot{r}$ is the specific growth rate:

$$
\begin{aligned}
& \dot{r}_{1}=\frac{E \dot{v} \mathcal{M}(L) / L-\left[\dot{p}_{M}\right] L^{3} / \kappa}{E+\left[E_{G}\right] L^{3} / \kappa} \\
& \dot{r}_{2}=\frac{E \dot{v} \mathcal{M}(L) / L-\left[\dot{p}_{M}\right] L^{3} / \kappa}{E+\kappa_{G}\left[E_{G}\right] L^{3} / \kappa}
\end{aligned}
$$

$\dot{p}_{C}$ is the mobilization flux:

$$
\dot{p}_{C}=E(\dot{v} \mathcal{M}(L) / L-\dot{r})
$$

$\dot{p}_{C m}$ is the mobilization flux assuming $f=1$ for an individual of structural length $L$ :

$$
\dot{p}_{C m}=\left[E_{m}\right] \frac{\left[E_{G}\right] \dot{v} \mathcal{M}(L) L^{2}+\left[\dot{p}_{M}\right] L^{3}}{\kappa\left[E_{m}\right]+\left[E_{G}\right]}
$$

$\mathcal{M}(L)$ is the shape correction function:

$$
\mathcal{M}(L)=\max \left(L_{b}, \min \left(L, L_{j}\right)\right) / L_{b}
$$

with $L_{b}$ and $L_{j}$ structural length at birth $\left(E_{H}=E_{H}^{b}\right)$ and metamorphosis $\left(E_{H}=E_{H}^{j}\right)$ respectively. 
Table B.2: Standard DEB model with V1-morphic juvenile I growth (as in Augustine et al., 2011a) further extended with reproduction buffer handling rules (as in Pecquerie et al., 2009), a toxico-kinetic module (as in Kooijman and van Haren, 1990) and degradation of structure to handle starvation (as in Augustine et al., 2011b). See text for addition specification of terms. The logical boolean, e.g. $(x<y)$, is enclosed in parentheses and has value 1 if true and value 0 if false.

For embryo and juveniles $\left(E_{H}<E_{H}^{p}\right)$ :

if $\kappa \dot{p}_{C} \geq\left[\dot{p}_{M}\right] L^{3} \quad \dot{r}=\dot{r}_{1} \quad$ else $\quad \dot{r}=\dot{r}_{2}$

For adults $\left(E_{H}=E_{H}^{p}\right)$ :

if $\kappa \dot{p}_{C} \geq\left[\dot{p}_{M}\right] L^{3}$

$\frac{d}{d t} E_{R}^{1}=\left(E_{R}^{0}>0\right)\left(t>t_{b}\right)\left(\kappa_{R}\left((1-\kappa) \dot{p}_{C m}-\dot{k}_{J} E_{H}^{p}\right)\right)$

$\frac{d}{d t} E_{R}^{0}=\left(\max \left(0,(1-\kappa) \dot{p}_{C}-\dot{k}_{J} E_{H}^{p}\right)-\frac{d}{d t} E_{R}^{1}\right)\left(E_{H}=E_{H}^{p}\right)$

$\dot{r}=\dot{r}_{1}$

else if $\kappa \dot{p}_{C}<\left[\dot{p}_{M}\right] L^{3}$

$\frac{d}{d t} E_{R}^{1}=0$

$\frac{d}{d t} E_{R}^{0}=\left(\max \left(0,(1-\kappa) \dot{p}_{C}-\dot{k}_{J} E_{H}^{p}\right)-\left(\left[\dot{p}_{M}\right] L^{3}-\kappa \dot{p}_{C}\right)\left(E_{R}^{0}>0\right)\right)\left(E_{H}=E_{H}^{p}\right)$

$\dot{r}=0 \quad$ if $\quad E_{R}^{0}>0 \quad$ and $\quad \dot{r}=\dot{r}_{2} \quad$ if $\quad E_{R}^{0}=0$

\section{Dynamics of state variables:}

$$
\begin{aligned}
& \frac{d}{d t} E=\dot{p}_{A}-\dot{p}_{C} \\
& \frac{d}{d t} L=\frac{\dot{r}}{3} L \\
& \frac{d}{d t} E_{H}=\max \left(0,\left((1-\kappa) \dot{p}_{C}-\dot{k}_{J} E_{H}\right)\right)\left(E_{H}<E_{H}^{p}\right) \\
& \frac{d}{d t} E_{R}=\frac{d}{d t} E_{R}^{0}+\frac{d}{d t} E_{R}^{1} \\
& \frac{d}{d t}\left[M_{Q}\right]=\frac{\dot{k}_{e}}{l}\left(P_{V d} c_{d}-\left[M_{Q}\right] P_{V W}\right)-\left[M_{Q}\right] \dot{r}
\end{aligned}
$$




\section{Initial conditions, ingestion level and toxicity param- eter values for all of the studies}

The initial amount of structure for each female in both the $37 \mathrm{~d}$ and $15 \mathrm{~d}$ reproduction trials are obtained from the final observed total length which are plotted in fig. C.2. The values of initial amount of reproduction buffer material, ingestion level, batch energetic density and day batch preparation is initiated for each female in both experiments is recorded in table C.3.

In addition to both reproduction trials three other studies were analysed. The values of initial conditions and ingestion level for each of the three studies as well as values of toxicity parameters for all of the studies are in table C.4.

Reserve dynamics are such that weak homeostasis is respected (Kooijman, 2010, Chap.2), i.e. under constant feeding conditions reserve density $[E]=E / L$ remains constant and reserve energy $E(t)$ can be computed as:

$$
E(t)=f\left[E_{m}\right] V(t)
$$

with $\left[E_{m}\right]=\left\{\dot{p}_{A m}\right\} / \dot{v}\left(\mathrm{~J} \mathrm{~cm}^{-3}\right)$ maximum reserve density and $\dot{v}\left(\mathrm{~cm} \mathrm{~d}^{-1}\right)$ reserve mobilisation (table 1). We make use of this property to compute initial amount of reserve $E(0)$ for each female in the two reproduction trials as well as a mean initial value for the two bioaccumulation studies (Barillet et al., 2005, 2011).

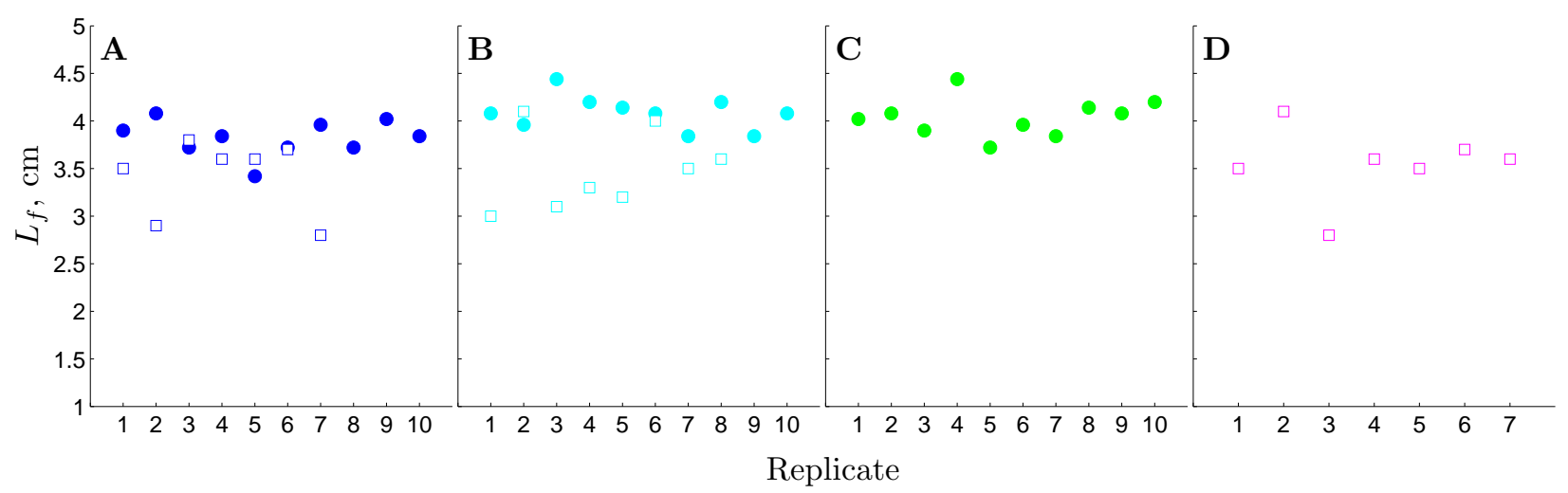

Figure C.2: Final observed length for each female in 37d (full circles) and 15d (square) reproduction trials. A: control; B: $c_{d}=84 \mathrm{nM}$; C: $c_{d}=420 \mathrm{nM}$; D: $c_{d}=1054 \mathrm{nM}$, with $c_{d}$ the nominal concentration of depleted uranium in holding tank water (nM); see fig. A.1, Appendix A for measured values. x-axis: replicate number with e.g. 1 designating female 1 . y-axis: total length $L_{f}$ (snout to end of caudal fin) $\mathrm{cm}$. The initial amount of structure for each replicate was computed as: $L(0)=0.94 \delta_{M} L_{f}$ (see text). Please note, females in the $15 \mathrm{~d}$ trial were on average smaller. 
Table C.3: Initial amount of reproduction buffer material and parameters for buffer handling rules for each female in the 37d (this study) and in the 15d (Bourrachot, 2009) reproduction trials. Initial structure $L(0)(\mathrm{cm})$ is assumed to be $L(0)=0.94 \delta_{M} L_{f}$, with $L_{f}$ final observed length (fig. C.2) and $\delta_{M}(-)$ the shape coefficient for adults (table 1$)$. Initial energy in reserve $(\mathrm{J})$ is computed using Eq. (C.6). $\left[E_{B}\right]\left(\mathrm{J} \mathrm{cm}^{-3}\right)$ : batch energy density; $E_{R}(0)(\mathrm{J})$ : initial amount of reproduction buffer material; $t_{b}$ day of experiment batch preparation is triggered during the $20 \mathrm{~d}$ accumulation phase which preceded the $15 \mathrm{~d}$ reproduction trial; $t>t_{b}$ for the $37 \mathrm{~d}$ experiment. For the sake of simplicity, we assume that $E_{R}(0)$ comprises only unripe buffer material.

\begin{tabular}{|c|c|c|c|c|c|c|c|}
\hline \multirow[b]{2}{*}{ Replicate } & \multicolumn{3}{|c|}{ This study (37 d trial) } & \multicolumn{4}{|c|}{ Bourrachot (2009) (14 d trial) } \\
\hline & $f(-)$ & $\begin{array}{l}{\left[E_{B}\right](\mathbf{J}} \\
\left.\mathbf{c m}^{-3}\right)\end{array}$ & $\begin{array}{l}E_{R}(0) \\
(\mathbf{J})\end{array}$ & $f$ & $\begin{array}{l}{\left[E_{B}\right](\mathbf{J}} \\
\left.\mathbf{c m}^{-3}\right)\end{array}$ & $E_{R}(0)(\mathbf{J})$ & $t_{b}(\mathbf{d})$ \\
\hline \multicolumn{8}{|l|}{ Control: } \\
\hline 1 & 0.76 & 3000 & 450 & 0.65 & 1200 & 0 & 13 \\
\hline 2 & 0.60 & 3000 & 718 & 0.72 & 1000 & 0 & 20 \\
\hline 3 & 0.75 & 3000 & 700 & 0.70 & 1000 & 0 & 15 \\
\hline 4 & 0.80 & 3000 & 700 & 0.66 & 1400 & 0 & 20 \\
\hline 5 & 0.94 & 8000 & 300 & 0.78 & 1500 & 100 & 0 \\
\hline 6 & 0.60 & 3000 & 745 & 0.73 & 1580 & 0 & 19 \\
\hline 7 & 0.66 & 3000 & 744 & 0.67 & 1800 & 400 & 0 \\
\hline 8 & 0.54 & 3000 & 734 & n.a. & n.a. & n.a. & n.a. \\
\hline 9 & 0.54 & 3000 & 736 & n.a. & n.a. & n.a. & n.a. \\
\hline 10 & 0.64 & 3000 & 713 & n.a. & n.a. & n.a. & n.a. \\
\hline \multicolumn{8}{|l|}{$84 \mathrm{nM}:$} \\
\hline 1 & 0.77 & 7000 & 350 & 0.71 & 2000 & 413 & 0 \\
\hline 2 & 0.82 & 1734 & 1000 & 0.63 & 1000 & 0 & 20 \\
\hline 3 & 0.82 & 1734 & 200 & 0.78 & 1000 & 50 & 0 \\
\hline 4 & 0.75 & 7000 & 350 & 0.70 & 1000 & 0 & 20 \\
\hline 5 & 0.77 & 3000 & 80 & 0.68 & 1000 & 0 & 20 \\
\hline 6 & 0.82 & 2200 & 100 & 0.67 & 1000 & 0 & 20 \\
\hline 7 & 0.82 & 2000 & 500 & 0.73 & 1446 & 0 & 7 \\
\hline 8 & 0.69 & 3000 & 50 & 0.72 & 1446 & 0 & 20 \\
\hline 9 & 0.80 & 3000 & 200 & n.a. & n.a. & n.a. & n.a. \\
\hline 10 & 0.88 & 2000 & 600 & n.a. & n.a. & n.a. & n.a. \\
\hline \multicolumn{8}{|c|}{$420(37 d)$ and $1054(15 d)$ nM: } \\
\hline 1 & 0.85 & 3000 & 1000 & 0.75 & 1000 & 0 & 20 \\
\hline 2 & 0.79 & 3000 & 350 & 0.76 & 1000 & 450 & 0 \\
\hline 3 & 0.85 & 1000 & 1800 & 0.76 & 1000 & 150 & 0 \\
\hline 4 & 0.84 & 1200 & 900 & 0.75 & 1000 & 150 & 20 \\
\hline 5 & 0.82 & 3000 & 350 & 0.74 & 1000 & 450 & 0 \\
\hline 6 & 0.73 & 3000 & 350 & 0.76 & 1000 & 0 & 20 \\
\hline 7 & 0.82 & 1000 & 350 & 0.73 & 1000 & 0 & 20 \\
\hline 8 & 0.81 & 3000 & 350 & n.a. & n.a. & n.a. & n.a. \\
\hline 9 & 0.76 & 3000 & 350 & n.a. & n.a. & n.a. & n.a. \\
\hline 10 & 0.81 & 3000 & 350 & n.a. & n.a. & n.a. & n.a. \\
\hline
\end{tabular}


Table C.4: Toxicity parameters and initial conditions used for modelling all of the data sets in this study. For the reproduction trials initial structural length $L(0)$ was computed for each female according to her final observe length; initial amount of energy in the reproduction buffer $E_{R}(0)$ was also estimated for each female. In this case we set '-' in the fields of $E_{R}(0)$ and $L(0)$ and refer the reader to table C.3 for the values for each individual. $E(0)$ is computed according to Eq. (C.6). U-84, U-420 and U-2100 refer to three exposure conditions in Barillet et al. (2005). Survival parameters are non applicable (n.a.) to adult data sets. Parameter definition and units are specified in table 1.

\begin{tabular}{|c|c|c|c|c|c|c|c|}
\hline \multirow[t]{2}{*}{ parameter } & \multirow{2}{*}{$\begin{array}{l}\text { this } \\
\text { study }\end{array}$} & \multirow{2}{*}{$\begin{array}{l}\text { Bourrachot } \\
(2009)\end{array}$} & \multicolumn{3}{|c|}{ Barillet et al. (2005) } & \multirow{2}{*}{$\begin{array}{c}\text { Barillet et al. } \\
\text { (2011) }\end{array}$} & \multirow{2}{*}{$\begin{array}{l}\text { Bourrachot } \\
\text { et al. (2008) }\end{array}$} \\
\hline & & & $\overline{\mathrm{U}-84}$ & U-420 & $\mathrm{U}-2100$ & & \\
\hline$\dot{k}_{e}$ & 0.003 & 0.003 & 0.003 & 0.003 & 0.003 & 0.003 & 0.003 \\
\hline$P_{V d}$ & 19.1 & 19.1 & 19.1 & 19.1 & 19.1 & 19.1 & 0.005 \\
\hline$P_{E V}$ & 13.1 & 13.1 & 13.1 & 13.1 & 13.1 & 13.1 & 13.1 \\
\hline$P_{E V}$ & 13.1 & 13.1 & 13.1 & 13.1 & 13.1 & 13.1 & 13.1 \\
\hline$\left[M_{Q 0}^{\mathrm{M}}\right]$ & 32 & 32 & 32 & 32 & 32 & 32 & 32 \\
\hline$\left[M_{Q T}^{\mathrm{M}}\right]$ & 28.9 & 28.9 & 28.9 & 28.9 & 28.9 & 28.9 & 28.9 \\
\hline$\left[M_{Q 0}^{G}\right]$ & 0 & 0 & 0 & 0 & 0 & 0 & 0 \\
\hline$\left[M_{Q T}^{\mathrm{G}}\right]$ & 0.211 & 0.211 & 0.211 & 0.211 & 0.211 & 0.211 & 0.211 \\
\hline$\left[M_{Q 0}^{\mathrm{S}}\right]$ & n.a. & n.a. & n.a. & n.a. & n.a. & n.a. & 0 \\
\hline$\dot{b}_{\dagger}$ & n.a. & n.a. & n.a. & n.a. & n.a. & n.a. & 0.375 \\
\hline$\dot{h}_{\text {acc }}$ & n.a. & n.a. & n.a. & n.a. & n.a. & n.a. & 0.02 \\
\hline$f$ & - & - & 0.7 & 0.85 & 0.85 & 0.7 & 0.3 \\
\hline$L(0)$ & - & - & 0.383 & 0.383 & 0.383 & 0.451 & 0 \\
\hline$E_{R}(0)$ & - & - & 250 & 250 & 250 & 250 & 0 \\
\hline$E_{H}(0)$ & $E_{H}^{p}$ & $E_{H}^{p}$ & $E_{H}^{p}$ & $E_{H}^{p}$ & $E_{H}^{p}$ & $E_{H}^{p}$ & 0 \\
\hline
\end{tabular}




\section{Additional model simulations for evaluating toxic mode of action on metabolism of zebrafish}

\section{D.1 Control model fit to U-84 female reproductive data}

Table D.5: The control model is fit to toxicity data. Parameters for buffer handling rules for each individual female exposed to $84 \mathrm{nmol} \mathrm{l}^{-1}$ in the $37 \mathrm{~d}$ reproduction trial. At time 0 fish are 0.94 their final length, and have $f\left[E_{m}\right] L^{3}$ joules in their reserve. We consider that batch preparation is already initiated at the onset of the trial. We also assume that $E_{R}(0)$ comprises only unripe batch material.

\begin{tabular}{cccc}
\hline Replicate & $f$ & {$\left[E_{B}\right]\left(\mathrm{J} \mathrm{cm}^{-3}\right)$} & $E_{R}(0)(\mathrm{J})$ \\
\hline 1 & 0.598 & 1500 & 737 \\
2 & 0.863 & 1500 & 728 \\
3 & 0.861 & 1756 & 690 \\
4 & 0.595 & 1677 & 712 \\
5 & 0.830 & 3000 & 0 \\
6 & 0.830 & 3000 & 400 \\
7 & 0.830 & 2000 & 600 \\
8 & 0.550 & 2000 & 300 \\
9 & 0.830 & 2000 & 300 \\
10 & 0.821 & 2022 & 311 \\
\hline
\end{tabular}




\section{Control model fits for $37 \mathrm{~d}-\mathrm{U} 84$}
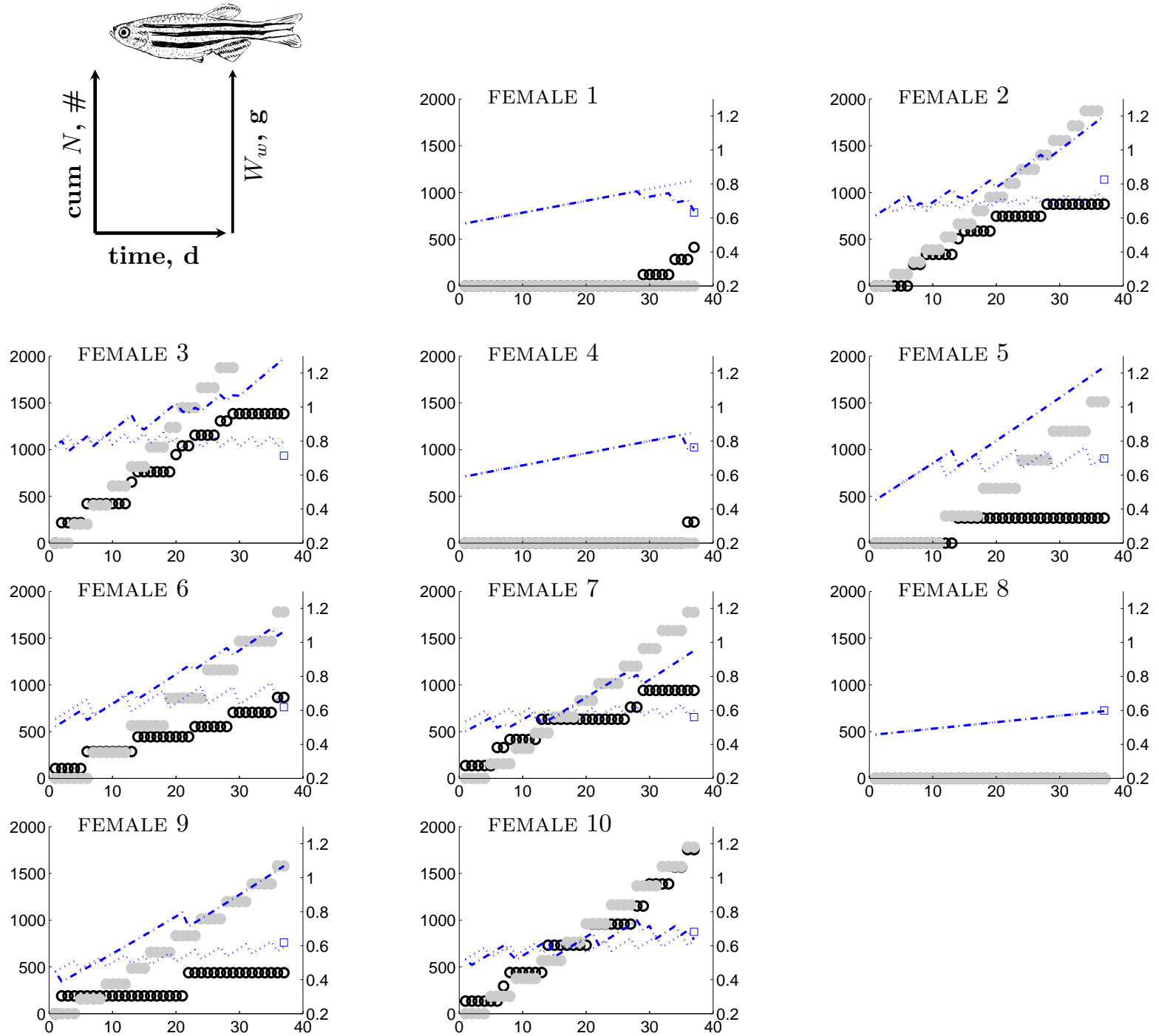

Figure D.3: Control model fits to individual females exposed to $84 \mathrm{nmol}^{-1}$. Left y-axis: empty circles (magenta) represent observed cumulated (cum) number of eggs spawned (\#) and full circles (red) represent model predictions. Right y-axis: dotted (blue) line represents predicted wet mass using buffer handling rules, mg. Dot-dashed (blue) line represents predicted wet mass without buffer handling rules, g. Physiological parameters for each female are in table D.5. The wet mass of the real number of eggs spawned are subtracted from the total predicted wet mass on each spawning day. See text for computations. The square (blue) represents final observed wet mass for that individual. 


\section{D.2 Initial amount of reserve in two females}

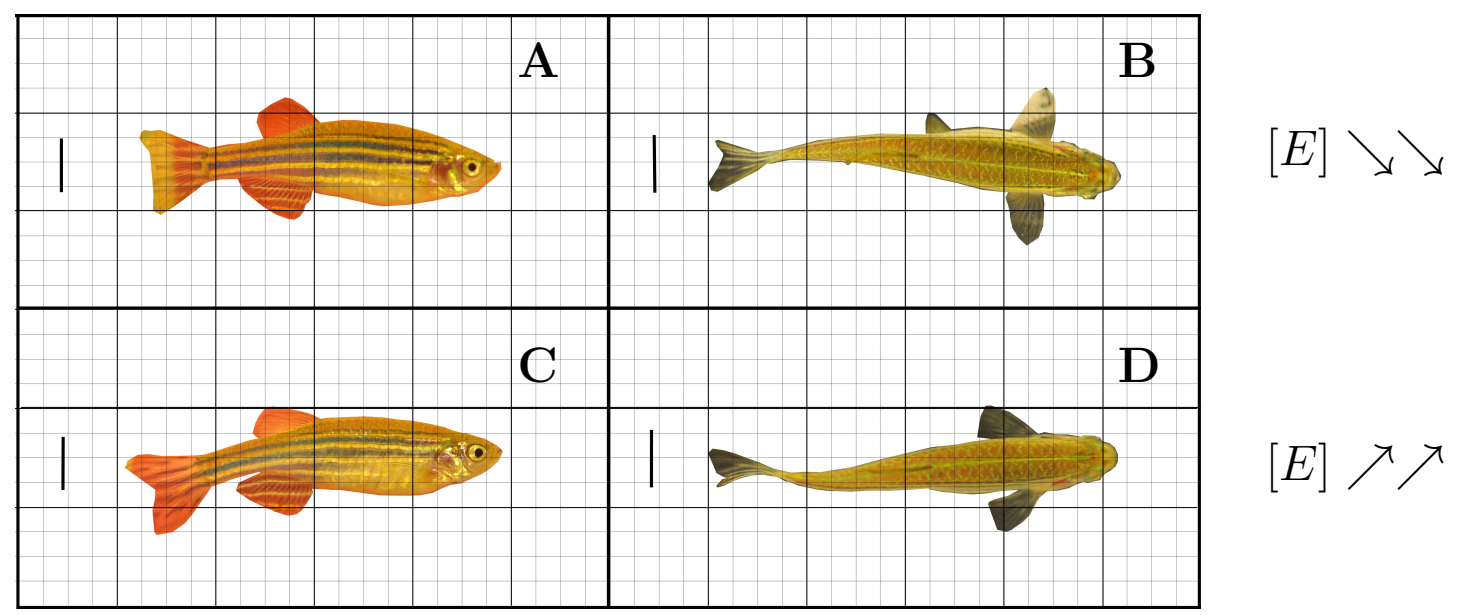

Figure D.4: Portraits taken during the third day of exposure to $420 \mathrm{nmol} \mathrm{U} \mathrm{l}^{-1}$. Lateral (A) and dorsal (B) portraits of Female 8. Lateral (C) and dorsal (D) portraits of female 10. Both females had quasi-identical final wet mass and length and both did not reproduce during the $37 \mathrm{~d}$ exposure (fig. C.2 and 6$)$. The difference between both females is that females 10 spawned 570 eggs $(\approx 280 \mathrm{mg}$ wet mass $)$ the day prior to exposure, i.e. 4 days before this photo was taken. Female 8 did not spawn at all during the 35 days acclimation. We suppose that while both female seem almost identical, female 10 must have started the experiment with a higher reserve density that female 8. Scalebar: 6mm. 


\section{D.3 Effects of increasing cost for synthesis of structure on adult reproductive output}

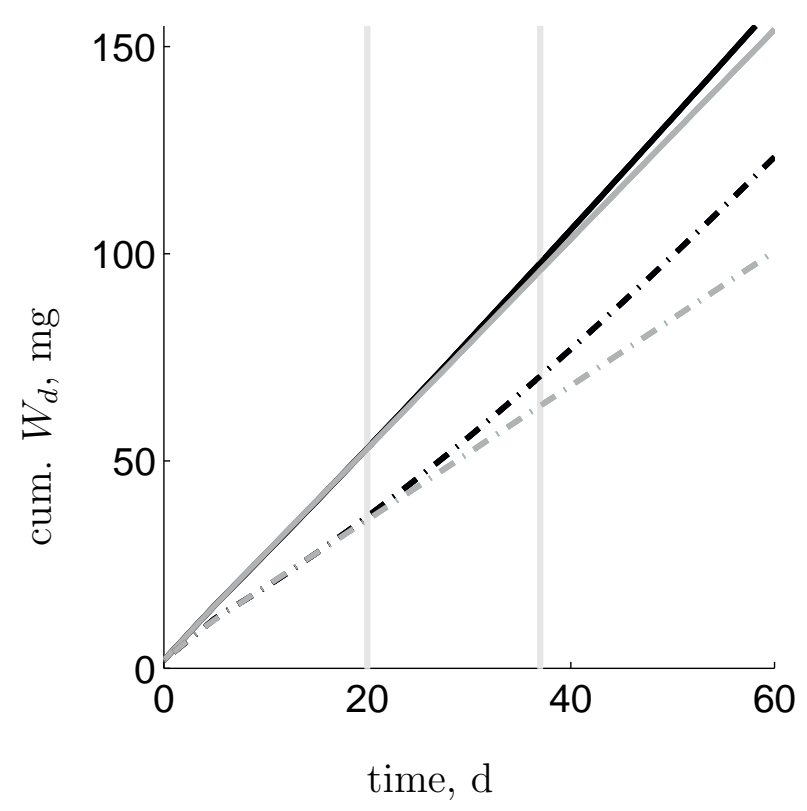

Figure D.5: DEB model simulation studies of mode of action $(\mathrm{G})$ on adult reproduction. $\mathrm{T}=25^{\circ} \mathrm{C}$, $f=0.75$. First horizontal light grey line: $20 \mathrm{~d}$; second horizontal light grey line: $37 \mathrm{~d}$. Cumulated (cum.) dry mass $W_{d}$ invested in reproduction, expressed as mg dry mass of reproduction buffer material, against time. Black: control; darker grey: $84 \mathrm{nM} \mathrm{U}$ in the water. The simulations use the same $\left[M_{Q 0}^{\mathrm{G}}\right]$ and $\left[M_{Q T}^{\mathrm{G}}\right]$ as that used for modelling embryo and early juvenile data sets (table C.4). Solid lines: individual of $3.7 \mathrm{~cm}$ total length (about the average total length of individuals in the $37 \mathrm{~d}$ reproduction trial); dot-dashed line: individual of $3.3 \mathrm{~cm}$ total length (about the average total length of individuals in the $15 \mathrm{~d}$ reproduction trial). 


\title{
E Model fits for individuals females in Bourrachot (2009)
}

\author{
15d-U0
}
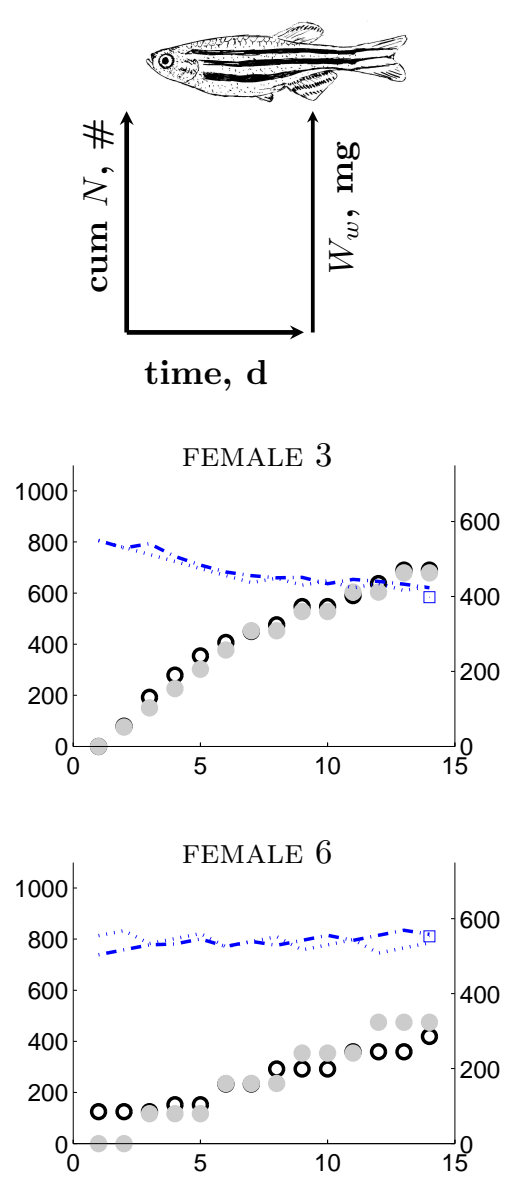
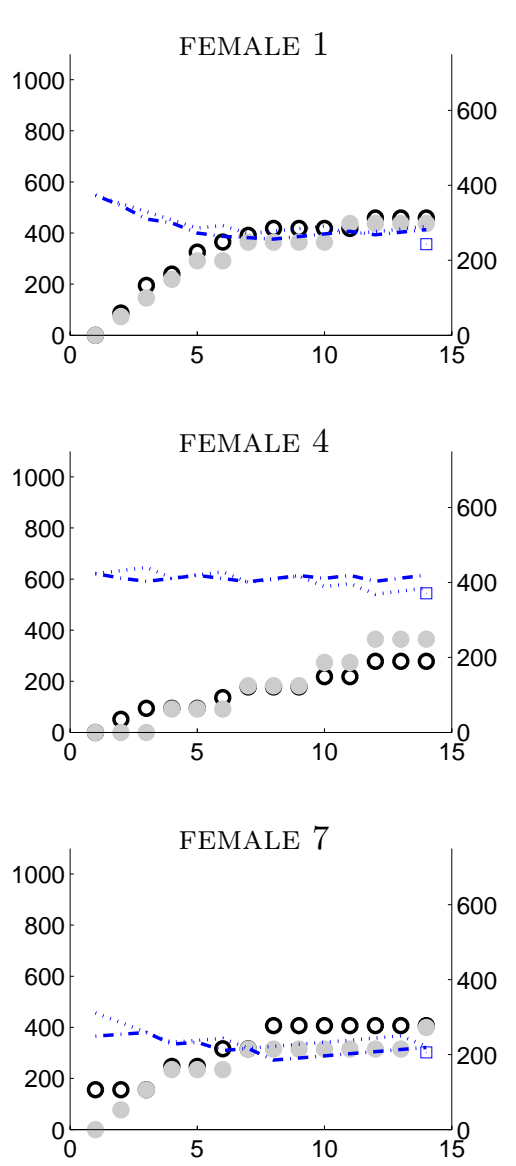
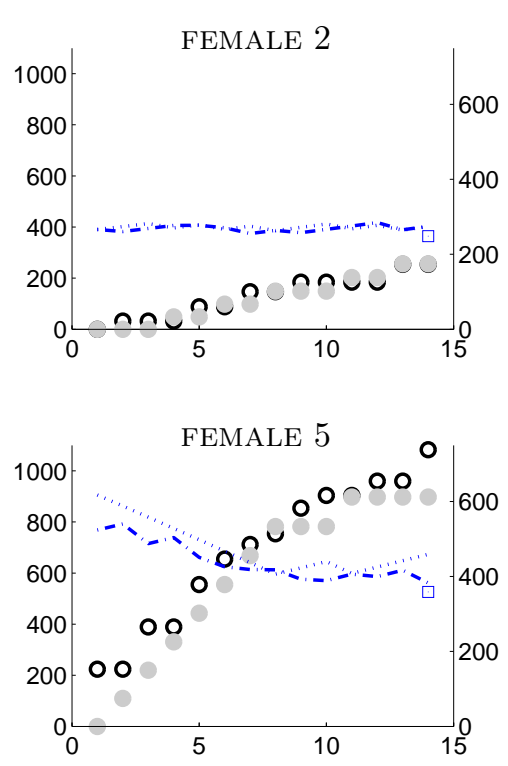

Figure E.6: DEB model fit to individual control females. Data kindly provided by S. Bourrachot from her thesis (Bourrachot, 2009). Parameters can be found in tables C.4 and C.3. Left y-axis: empty circles (black) represent observed cumulated (cum) number $N$ of eggs spawned (\#) and full circles (grey) represent model predictions. Right y-axis: dotted (blue) line represents predicted wet mass using buffer handling rules, mg (eq. (7)). Dot-dashed (blue) line represents predicted wet mass without buffer handling rules, mg (eq. (8)). The square (blue) represents final observed wet mass for that individual. Initial conditions, batch energetic density and ingestion level for each female are recorded in table C.3, Appendix C. 


\section{5d-U84}
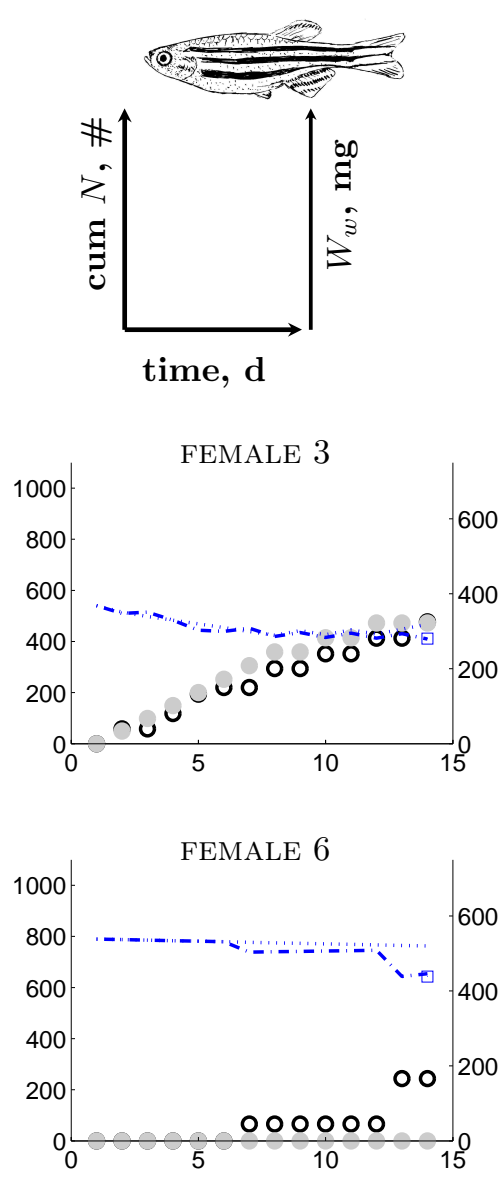
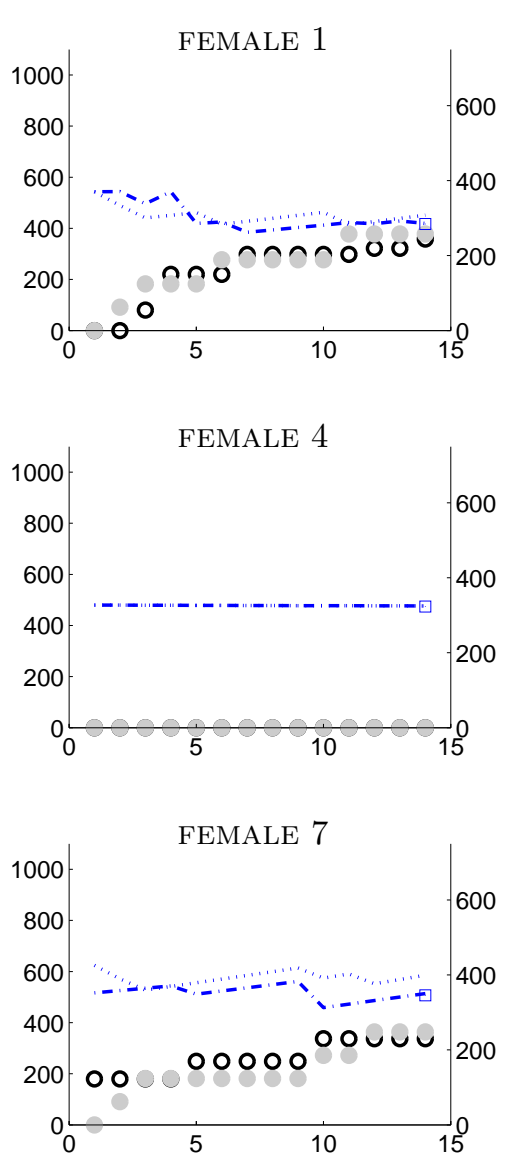

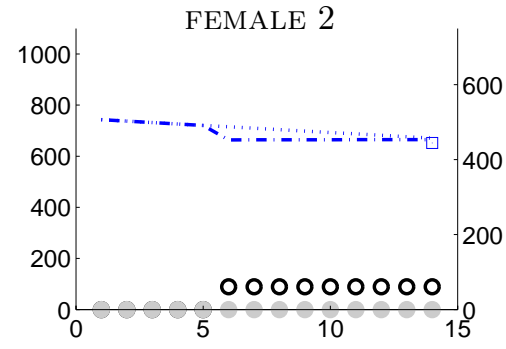

FEMALE 5

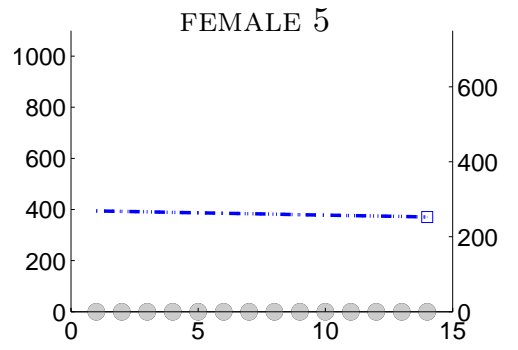

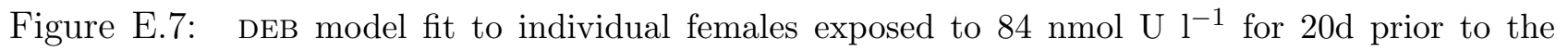
reproduction trial. Data kindly provided by S. Bourrachot from her thesis (Bourrachot, 2009). The model assumes U increases volume-linked somatic maintenance. Parameters can be found in tables C.4 and C.3. Left y-axis: empty circles (black) represent observed cumulated (cum) number $N$ of eggs spawned (\#) and full circles (grey) represent model predictions. Right y-axis: dotted (blue) line represents predicted wet mass using buffer handling rules, mg (eq. 7). Dot-dashed (blue) line represents predicted wet mass without buffer handling rules, mg (eq. (8)). The square (blue) represents final observed wet mass for that individual. Initial conditions, batch energetic density and ingestion level for each female are recorded in table C.3, Appendix (C). 


\section{5d-U1054}
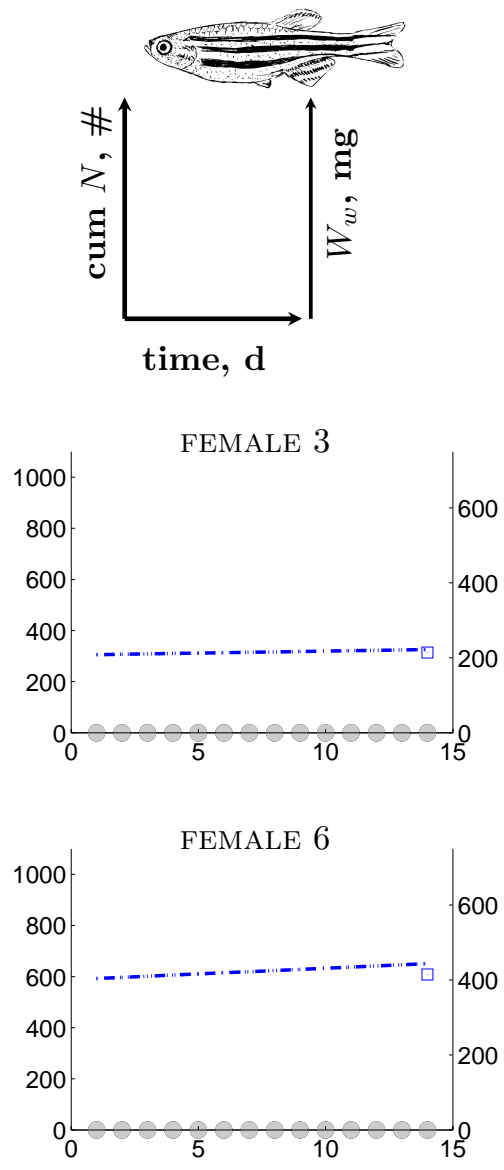
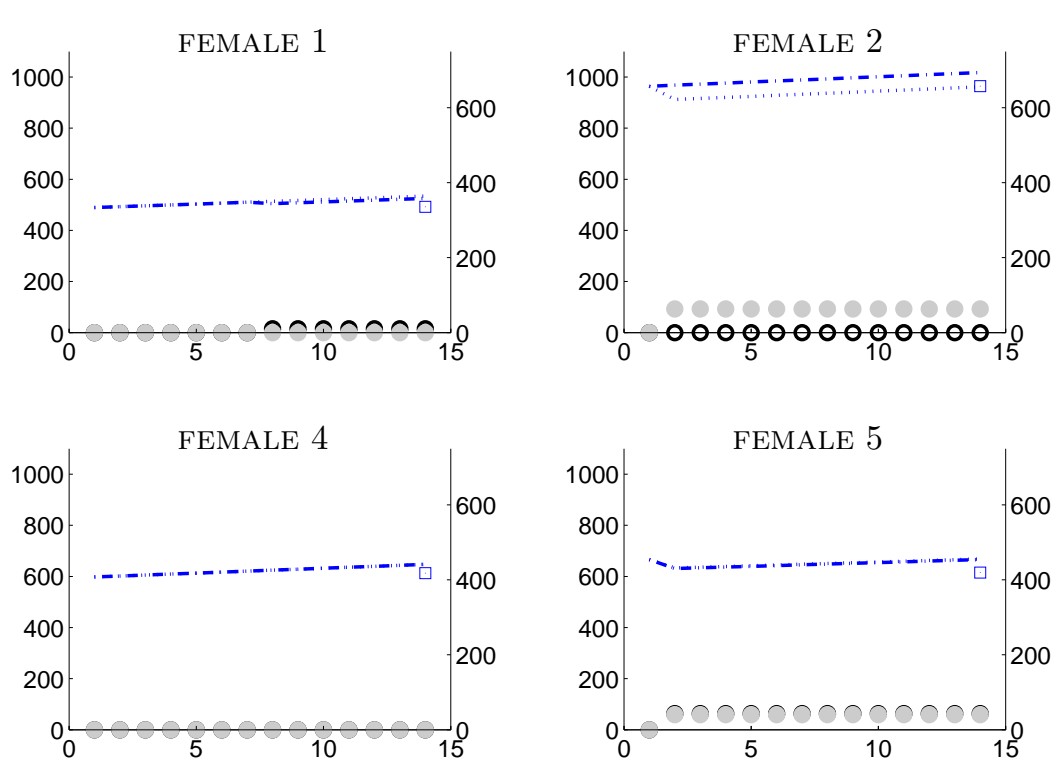

Figure E.8: DEB model fit to individual females exposed to $1054 \mathrm{nmol} \mathrm{U}^{-1}$ for $20 \mathrm{~d}$ prior to the reproduction trial. Data kindly provided by S. Bourrachot from her thesis (Bourrachot, 2009). The model assumes $U$ increases volume-linked somatic maintenance. Parameters can be found in tables C.4 and C.3. Left y-axis: empty circles (black) represent observed cumulated (cum) number $N$ of eggs spawned (\#) and full circles (grey) represent model predictions. Right y-axis: dotted (blue) line represents predicted wet mass using buffer handling rules, mg (eq. (7)). Dot-dashed (blue) line represents predicted wet mass without buffer handling rules, mg (eq. (8)). The square (blue) represents final observed wet mass for that individual. Initial conditions, batch energetic density and ingestion level for each female are recorded in table C.3, Appendix C. 